\title{
Evolución paleoambiental de la diatomita de Agostitlán, Michoacán, durante la transición Pleistoceno-Holoceno
}

\section{Paleoenvironmental evolution of the Agostitlán diatomite, Michoacán, during the Pleistocene- Holocene transition}

\author{
Ma. de Jesús Sánchez-González ${ }^{1, *}$, Isabel Israde Alcántara², Juan Julio Morales ${ }^{3}$, \\ Avto Goguitchaichvili ${ }^{1}$
}

${ }^{1}$ Doctorado en Ciencias de la Tierra, Instituto de Geofísica Universidad Nacional Autónoma de México. Ciudad Universitaria, 04510 Coyoacán, CDMX, México.

${ }^{2}$ Instituto de Investigaciones en Ciencias de la Tierra (INICIT), Universidad Michoacana de San Nicolás de Hidalgo. Avenida Francisco J. Múgica S/N, Edificio U-4 Ciudad Universitaria, 58030, Morelia, Michoacán, México.

${ }^{3}$ Laboratorio Interinstitucional de Magnetismo Natural (LIMNA), Unidad Michoacán del Instituto Geofísica, Universidad Nacional Autónoma de México, Antigua Carretera a Pátzcuaro No. 8701, Col. Ex Hacienda de San José de la Huerta, 58190, Morelia, Michoacán, México.

* Autor para correspondencia:

(M.J. Sánchez-González) isaisrade@gmail.com

\begin{abstract}
Gómo citar este artículo:
Sánchez-González, M.J., Israde Alcántara, I., Morales, J.J., Goguitchaichvili, A., 2021, Evolución paleoambiental de la diatomita de Agostitlán, Michoacán, durante la transición Pleistoceno-Holoceno: Boletín de la Sociedad Geológica Mexicana, 73 (1), A080620. http://dx.doi.org/10.18268/ BSGM202lv73nla080620
\end{abstract}

Manuscrito recibido: 23 de Agosto de 2019 Manuscrito corregido: 20 de Noviembre de 2019 Manuscrito aceptado: 1 de Diciembre de 2019

La revisión por pares es responsabilidad de la Universidad Nacional Autónoma de México.

Este es un artículo Open Access bajo la licencia CC $Y-N C$-SA (https://creativecommons.org/licenses/by-nc-sa/4.0/)

\section{RESUMEN}

El graben de Chapala-Acambay se caracteriza por el registro de yacimientos de diatomitas del Cuaternario con espesores variables, donde domina flora indicativa de lagos profundos. El depósito de diatomita de $6.70 \mathrm{~m}$ de espesor de la cuenca de represamiento de Agostitlán contiene un registro paleoambiental que marca la transición de condiciones menos frías de hace ca. 37,000 años cal aP hasta el inicio del Glacial tardío ca. 25,000 años cal aP. En el inicio de la formación del represamiento se desarrolló un cuerpo de agua profundo con dominio de Stephanodiscus niagarae, con escaso aporte de material detrítico dominando las facies arcillosas. Los valores de susceptibilidad magnética en este primer episodio del lago son bajos, con un par de notorios incrementos en la susceptibilidad magnética y asociados a periodos de erosión de las sierras altas que limitan la cuenca. Sucesivamente, hacia las condiciones del lago se vuelven fluctuantes; las facies sedimentarias son más detríticas, alternando con episodios de sedimentación fina, disminuyen las agrupaciones de Stephanodiscus spp y aumentan los porcentajes de Aulacoseira ambigua y Fragilaria spp, lo que sugiere condiciones más frías y alcalinas. La cima de la sucesión se caracteriza por un aumento en la concentración de los minerales magnéticos, así como por un descenso significativo de las especies planctónicas y ticoplanctónicas que vienen sustituidas por formas bentónicas y perifíticas, lo cual se interpreta como una respuesta al incremento de la aridez en esta zona, previo al inicio del Último Máximo Glacial.

Palabras clave: Diatomitas, México Central, Magnetismo Ambiental, MIS3, Wisconsin tardío.

\section{ABSTRACT}

The Chapala Acambay graben is characterized by several Quaternary diatomite deposits of variable thickness where dominates flora indicative of ancient deep lakes. The $6.70 \mathrm{~m}$ thickness diatomite-deposit of the Agostitlan dammed basin contains the paleoenvironmental record that show the transition from less cold conditions of 37,000 yr cal AP until the onset of the late Glacial period (ca. 25,000 yr cal AP). At the beginning of the lake formation a deep-water body was developed, characterized mostly by Stephanodiscus niagarae with little contribution of detrital material, mostly composed of argillaceous lithology. The magnetic susceptibility values in this first episode of the lake are relatively low, at least in the first two meters, with two notorious maxima associated with periods of erosion of the high-mountain ranges. Successively towards the Early Glacier the lake conditions become fluctuating; the sedimentation is more detrital, alternating with episodes of fine sedimentation in which the lowest values of magnetic susceptibility are revealed, which are accompanied by the decrease of Stephanodiscus spp communities and an increase of Aulacoseira ambigua and Fragilaria spp. This indicates an environment of dominantly cold and alkaline conditions. The top of the succession is characterized by an increase in the concentration of magnetic minerals, as well as by a significantly decrease of planktonic that becomes substituted by littoral benthic and periphytic species, which are interpreted as the response to the increase in aridity in this area at the beginning of the Last Glacial Maximum.

Keyreords: Diatomitas, Central Mexico, Environmental Magnetism, MIS3, late Wisconsin. 


\section{Introducción}

Una de las fuentes de información más atractivas para la reconstrucción paleoclimática son los sedimentos lacustres, debido a que los lagos son trampas naturales de acumulación continua de sedimentos. En nuestro país (Miranda et al., 1994, Mercer et al., 2014), y en otros como Perú (Fourtanier et al., 1993), Rusia (Khursevich et al., 2003), Estados Unidos (Bradbury y Krebs, 1982) y Francia (Seryeissol y Gasse, 1991), se observan grandes acumulaciones de diatomita cuya formación está ligada directamente al contexto de grabens en ambientes volcánicos. El tiempo en que se depositaron estos yacimientos fue durante el Neógeno tardío-Cuaternario temprano (Israde et al., 2010a), en el contexto de un clima húmedo dominante. La apertura de las cuencas dio lugar a un fallamiento con subsidencia y a la emisión de grandes cantidades de ceniza volcánica, que proveyeron la sílice necesaria para la formación de las diatomitas (Israde y Garduño 1999).

El clima ha jugado un papel importante, ya que, durante transgresiones (etapas de aumento en el nivel lacustre), se generaron sistemas lacustres profundos. Por el contrario, durante las regresiones (etapas del descenso del nivel lacustre) y, con escasa humedad, tuvieron lugar lagos someros.

Los lagos suponen un repositorio natural de información ambiental, puesto que los materiales que se depositan en ellos provienen de la atmósfera, la escorrentía o los procesos diagenéticos y biogénicos que tienen lugar en el mismo (dependiendo del tipo de cuenca lacustre, características del lago, entre otros factores). Asimismo, los procesos diagenéticos, debidos a las características fisicoquímicas del fondo del lago, pueden disolver y/o precipitar minerales (entre ellos los ferromagnéticos), ya que son muy sensibles a los cambios en las condiciones óxido-reducción (Vázquez, 2004). Numerosas investigaciones han tratado de establecer las relaciones entre las variaciones climáticas ocurridas durante el Cuaternario y la mineralogía magnética de suelos y paleosuelos de diversas localidades en el mundo, (Liu et al., 2007;
Jordanova et al., 2003; Bidegain et al., 2009; Geiss et al., 2008; Geiss y Zanner, 2006) así como con la contaminación (Orgeira et al., 2008).

En sedimentos lacustres los análisis magnéticos se han realizado de forma extensiva desde 1975 Jelonowsky et al., 1997, Van Vugt et al., 1998, Maher y Thompson, 1999), aunque en México son escasos los estudios donde se analizan en profundidad las propiedades magnéticas en ellos: en la Cuenca de México (Ortega et al., 2000), el alto Lerma (Caballero et al., 2002), Zacapu (Ortega et al., 2002), Pátzcuaro (Fisher et al., 2003), Zirahuén (Vázquez et al., 2010), el desierto de Sonora (Ortega et al., 1999), el desierto de Chihuahua (Urrutia et al., 1997), en Nayarit (Vázquez, 2004), y, recientemente, en Chapala (Morales et al., 2019). Sin embargo, ninguna investigación en México se ha llevado a cabo en diatomitas aplicando técnicas novedosas de magnetismo ambiental.

Éste relaciona la mineralogía magnética con diversos procesos ambientales a través del análisis de las propiedades magnéticas de los materiales geológicos formados bajo éstos y su comparación con características físicas, químicas y biológicas. La adquisición de remanencia magnética en minerales, principalmente óxidos de hierro y sulfuros, se produce de manera ubicua en sedimentos, los cuales pueden actuar como registradores sensibles de los climas del pasado porque, a medida que el clima ha variado (glacial - interglacial), la mineralogía, el estado de dominio magnético, la composición y la fuente de estos minerales ha cambiado también (Ortega et al., 2000). Muchos estudios de este tipo se basan únicamente en la susceptibilidad magnética como indicador (o proxy) de las variaciones climáticas y ambientales. No obstante, ya que este parámetro depende no sólo de la concentración de partículas ferrimagnéticas, sino también de la presencia de un amplio rango de minerales magnéticos y de sus distribuciones de tamaño (Geiss y Zanner, 2006), la interpretación de la $x$ rara vez es única y directa, por lo que las conclusiones pueden verse comprometidas si no se han caracterizado adecuadamente los componentes magnéticos. Por ello, los análisis de magnetismo deben involucrar técnicas 
que determinen la composición, concentración y distribución de tamaños de dominio de los minerales magnéticos, y, frecuentemente, requieren de información adicional de otros parámetros no magnéticos para lograr una acertada interpretación paleoambiental. Gran parte de las secuencias lacustres se caracterizan por registrar variaciones en $x$. Un aumento de este parámetro se debe, en la mayoría de los casos, a un crecimiento de los minerales ferrimagnéticos (magnetita y/o maghemita) de tamaño ultrafino superparamagnético (SP, < $0.01 \mu \mathrm{m})$ a dominio sencillo (DS, < $0.1 \mu \mathrm{m}$; por ejemplo, Maher, 1999; Hunt et al., 1995), pero también al incremento de partículas tanto ferrimagnéticas como antiferrimagnéticas (hematita o goethita) (Geiss et al., 2004). El origen de dicho incremento se ha interpretado de diferentes maneras, entre ellas la ocurrencia de incendios, fermentación, calentamiento, actividad biológica (Tite y Linington, 1975; Mullins, 1977; Maher, 1986; Thompson y Oldfield, 1986), la contaminación del suelo (Chaparro, 2006) y la transformación de minerales paramagnéticos o antiferrimagnéticos a fases ferrimagnéticas (Geiss y Zanner, 2006).

La magnoestratigrafía aplicada a secuencias lacustres con depósitos volcánicos intercalados ha sido utilizada a fin de asociarlos a parámetros orbitales reconocidos, sobre todo en diatomitas neógenas (Van Vugt et al., 1998, Deino et al., 2006).

En este trabajo se pretende conocer la cronología de la diatomita de Agostitlán y las aplicaciones del análisis de parámetros magnéticos para la reconstrucción paleoambiental. Este registro es de interés ya que fue la última diatomita que se desarrolló en el margen suroeste del graben por efecto de represamiento al final de Wisconsin. La aplicación del magnetismo ambiental en la diatomita de Agostitlán es de relevancia dada su pureza, por lo que se puede inferir la utilidad de aplicar este proxy para distinguir fases lacustres.

Asimismo, se presenta el análisis de las propiedades magnéticas y su utilidad en el análisis de la reconstrucción de la evolución paleoambiental de una diatomita depositada durante el Pleistoceno en la región de Agostitlán, Michoacán.

\section{Marco geológico}

La localidad de estudio se encuentra en el Centro occidente de México, donde, desde el Paleoceno al Cuaternario, la fisiografia se ha transformado como resultado de la compleja evolución geodinámica del margen del Pacífico y la fragmentación de la placa de América del Norte (Aranda-Gómez et al., 2005). Las principales provincias fisiográficas en el centro de México están constituidas por Cuencas, Cordilleras y depresiones tectónicas de orientación N-S y NNO-SSE (Aranda-Gómez et al., 2005) y la Sierra Madre Oriental. Esta última provincia, junto con la Miocénica de la Sierra Madre Occidental y el Altiplano central Mioceno, constituyen los límites del cinturón Volcánico Transmexicano (CVTM), cuya principal actividad del CVTM se desarrolló después de 12 Ma en la región centro-occidental (Garduño-Monroy y Gutierrez-Negrín, 1992). El paisaje de la región está caracterizado por grandes edificios volcánicos y hacia el norte la fragmentación genera fosas y semigrabens como productos de un régimen transtensivo NO-SE, durante el Mioceno-Plioceno formando un punto triple. El brazo E-O del punto triple constituye el denominado Graben ChapalaAcambay, localizado en el Centro-Occidente de México (Garduño et al., 1993), desde los márgenes Oeste del Lago de Chapala en Jalisco hasta la población de Acambay, Estado de México (Figura 1). Dentro del Graben se han establecido cadenas de lagos donde la actividad volcánica y climática propició el depósito de hasta 50 metros de diatomitas laminares, destacando las de Acambay, las cuales están muy tectonizadas (Rodríguez- Pascua et al., 2010).

Durante el Plioceno-Holoceno el magmatismo se convirtió de carácter monogenético, en la construcción del moderno Cinturón Volcánico TransMexicano. La actividad volcánica, junto con la reactivación de las fallas NO-SE y NE-SO y las emisiones piroclásticas de las calderas de Amealco, Azufres, Huichapan y La Primavera, contribuyó al relleno sedimentario lacustre y fluvial con depósitos aluviales intercalados. 
El río Lerma, el cual drena casi a todo lo largo del Graben, es otra característica determinante en la configuración fisiográfica del centro de México. Las estructuras trans-tensionales, junto con los edificios volcánicos y lava emitidos, controlaron los límites del río Lerma y de los grandes lagos y ha sido importante en su permanencia de los lagos más extensos durante el Neógeno-Cuaternario (Israde et al., 2010b).

No existen investigaciones de detalle en la zona, sin embargo, en la Carta Geológica de Michoacán se observa que el basamento de la zona de Agostitán está asociado a lavas de edad Mio-Pliocénica, de composición basáltica a andesítica con afinidad calcoalcalina, alternando con brechas volcánicas, escorias y productos piroclásticos que muestran alteración evidente (Garduño et al.,1993). La pequeña cuenca fue represada por la colada de un estratovolcán en su margen oriente y, sucesivamente, cubierta de forma parcial por depósitos fluviales y aluviales, así como por un lahar proveniente del occidente. Dicha cuenca está limitada, morfológicamente, por una falla Norte Sur que corre a lo largo de Jungapeo hasta Agostitlán.
Los depósitos lacustres presentan cierta pureza y su base ha sido fechada en 32,540 +/- 180 c14 aP (Pleistoceno superior). Su cima viene cubierta por paleosuelos cuyo contacto con la diatomita fue fechado en 20,950 +/- 70 BP c14 AaP. El intervalo de tiempo en el que se desarrolló la diatomita se enmarca dentro del Wisconsin tardío (Moran y Clayton, 1984) y el último Máximo Glaciar (Clark et al., 2009, Lambeck et al., 2014).

La diatomita de Agostitlán (Figura 2) está ubicada en el sector meridional del estado de Michoacán (19³2’08.96” N y 100²9’31.99 O a una altitud de 1711 m.s.n.m.). Se inserta dentro de un graben orientado NO-SE que se alinea con la caldera de los Azufres. Este graben NO-SE corta las estructuras E-O que limitan el lago de Chapala y Cuitzeo, los dos lagos activos más grandes de México, así como el extinto lago de Acambay. En los límites de éstos afloran diatomitas asignadas al Neógeno-Guaternario (Israde et al., 2010b), lo que permite la reconstrucción de la evolución y el ambiente en el que se desarrolló la abundante fauna de vertebrados que vivió en el entorno de ellos (Miller Wade y Carranza-Castañeda, 1984; Mercer et al., 2014).

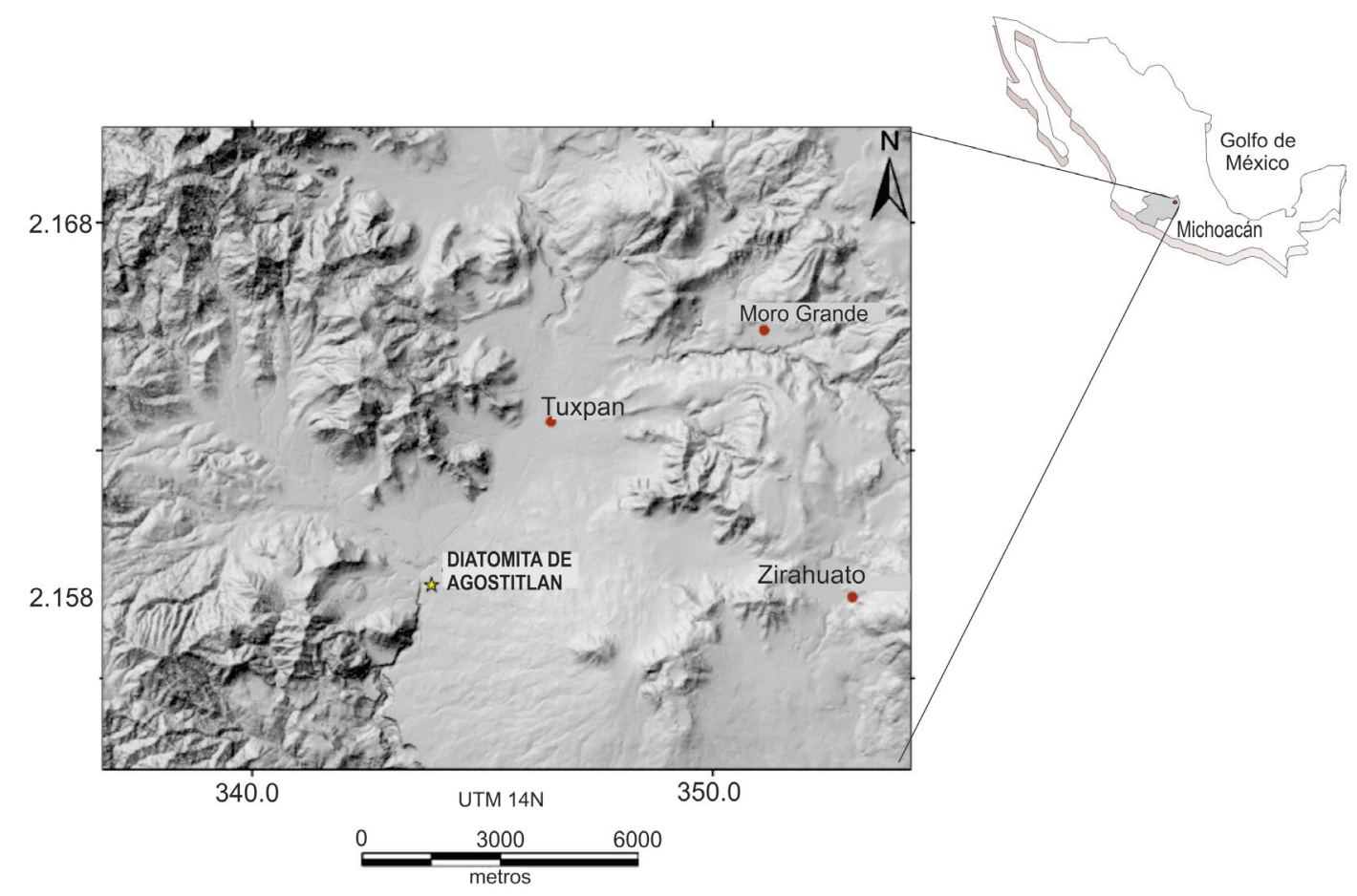

Figura 1 Ubicación geográfica del sitio de muestreo y modelo digital de elevación. 
$103^{\circ} 00^{\prime}$

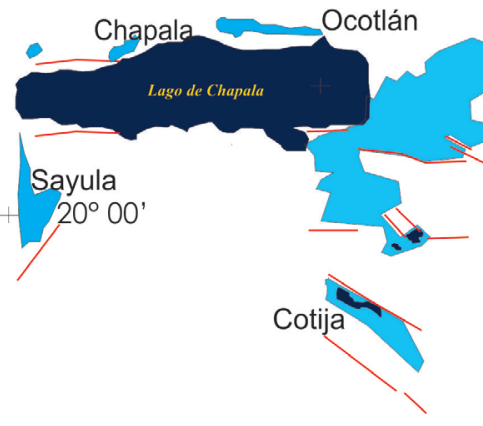

$+19^{\circ} 30^{\prime}$ $102^{\circ} 00^{\prime}$

Graben de

Penjamillo

Zamora

Corredor Tarasco

Patamban

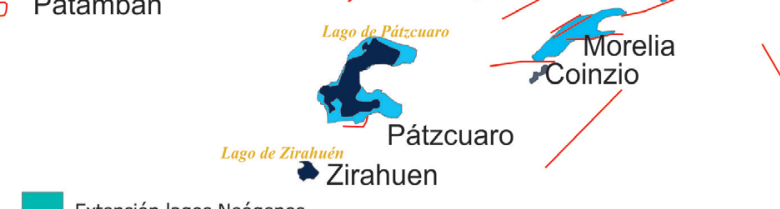

Extensión lagos Neógenos

Lagos actuales

Fallas y lineamientos

Figura 2 Brazo Este del punto triple denominado Graben Chapala-Acambay. En amarillo localización de la diatomita de Agostitlán. Imagen modificada de Israde-Alcántara et al, 2010b.

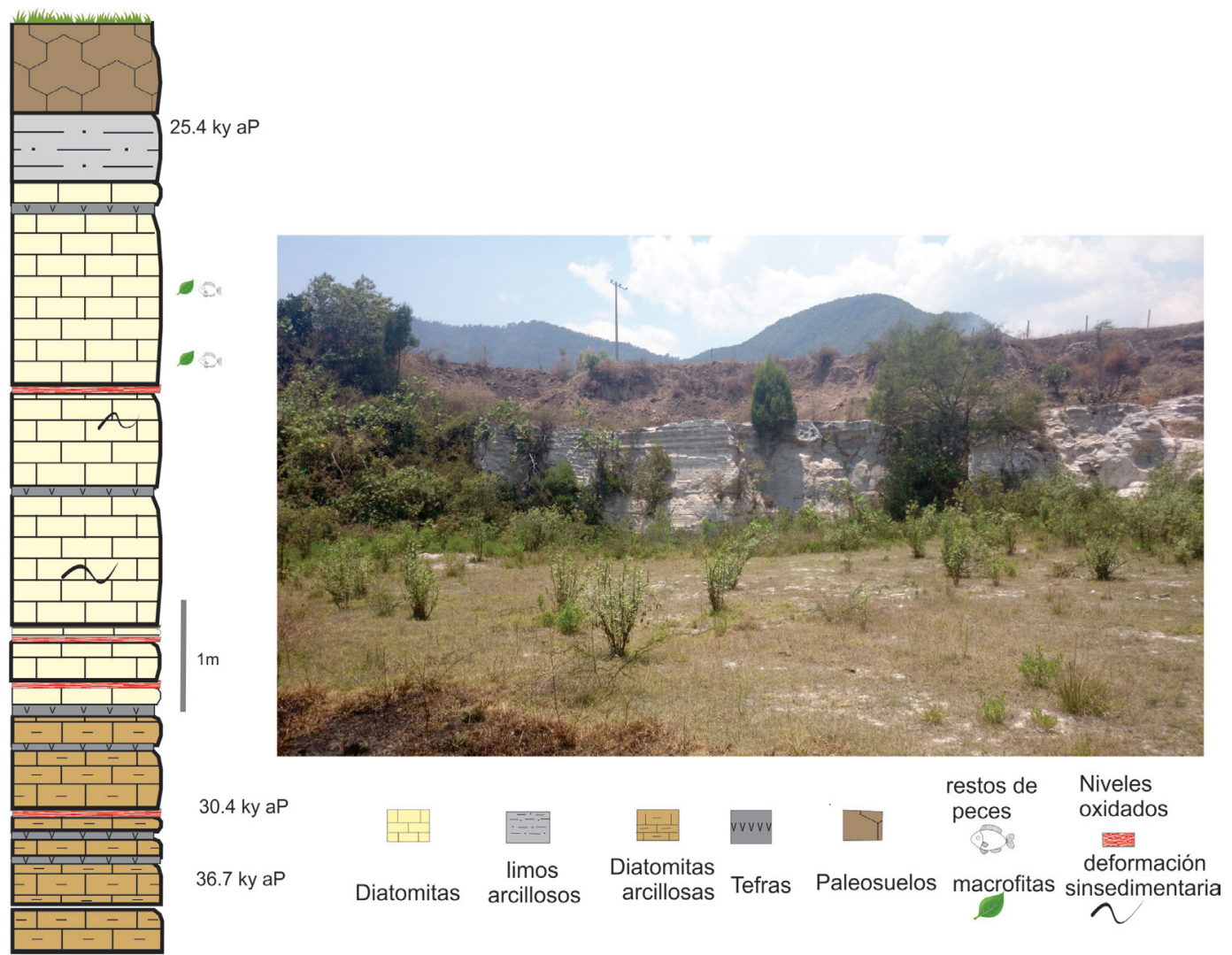

Figura 3 Columna estratigráfica del sector de más alta pureza de la diatomita muestreada e imagen del afloramiento. 


\section{Material y métodos}

Se muestreó la columna estratigráfica de $\sim 7 \mathrm{~m}$ (19²'08.96” N y 100²9’31.99 O, a 1711 msnm), obteniéndose 67 muestras, de la base hacia la cima a cada $10 \mathrm{~cm}$. Para su análisis de diatomeas se siguió el método propuesto por Gasse (1980), que consiste en pesar $0.5 \mathrm{~g}$ de muestra, la cual fue calentada con $\mathrm{HCl}$ al $10 \%$ y $\mathrm{H}_{2} \mathrm{O}_{2}$ al $30 \%$ para la eliminación de carbonatos y materia orgánica, respectivamente. Se enjuagó la muestra en varias ocasiones hasta que esta alcanzó un $\mathrm{pH}$ neutro. Las suspensiones purificadas y diluidas en $100 \mathrm{ml}$ se colocaron en portaobjetos y se montaron en una resina con un alto índice de refracción (náfrax I.R. 1.73+). La observación de las diatomeas se llevó a cabo en un microscopio óptico OLYMPUS BYMAX 50 con una cámara integrada Modelo DP12 a 1000x. Las imágenes editadas fueron clasificadas mediante la comparación con bibliografia especializada (Gasse, 1980; Germain, 1981; Krammer, 1980; Krammer y Lange Bertalot, 1985; 1986; 1988; 1991a; 1991b; Fourtanier y Gasse, 1988; Serieyssol y Gasse, 1991; Khursevich, et al., 2002, 2003; Khursevich y Kociolek, 2012) e inventarios taxonómicos de Alga Base (Guiry y Guiry, 2015).

El conteo en cada laminilla se realizó por campos de observación, que comprende el área a partir del campo visual a 100x. Se utilizó aceite de inmersión para cada laminilla. Los conteos de las valvas se realizaron en transectos de $18 \mathrm{~mm}$ para cada muestra (18 mm en cubreobjetos cuadrado). Para calcular las abundancias relativas se aplicó la siguiente fórmula:

$$
\sum A B U N D=C_{t}\left(\frac{A}{a}\right)\left(\frac{V_{t}}{V_{p p}}\right)\left(\frac{1}{x}\right)
$$

Donde:

$\Sigma A B U N D=$ Abundancia relativa.

$\mathrm{C} t=$ Frústulas contadas en la laminilla.

$\mathrm{A}=$ Área de la laminilla.

$\mathrm{a}=$ Campo del microscopio $(0.18 \mathrm{~mm})$ por la distancia contada (18 mm).

$\mathrm{V} t=\mathrm{La}$ dilución de donde se tomó la muestra.

$\mathrm{V} p p=$ Microlitros que se colocaron sobre el cubreobjetos en el desecador $(0.2 \mathrm{ml})$.

$1=$ Constante.

$x=$ Peso de la muestra seca $(0.5 \mathrm{gr})$.

La abundancia relativa obtenida se expresó en $10^{7}$ valvas/gramo de sedimentos secos.

El muestreo de la columna sedimentaria de diatomita para análisis de magnetismo ambiental se realizó en cubos de acrílico de $\sim 8 \mathrm{~cm}^{3}$, los cuales se caracterizaron desde el punto de vista magnético: susceptibilidad magnética $(x)$, magnetización remanente natural (MRN o NRM, por sus siglas en inglés), magnetización remanente anhistérica (MRA) y magnetización remanente isotermal (MRI).

La susceptibilidad magnética en baja ( $x_{\mathrm{r}}$, $0.465 \mathrm{kHz})$ y alta frecuencia $\left(x_{\mathrm{hr}}, 4.65 \mathrm{kHz}\right)$ de las muestras discretas (cubos de $8 \mathrm{~cm}^{3}$ ) se midió por medio de un susceptibilímetro Bartington MS2B. Asimismo, se midió la susceptibilidad magnética con el equipo AGICO MFK-B, que trabaja a una sola frecuencia $(972 \mathrm{~Hz})$, como medio de verificación.

La intensidad de la magnetización en los diferentes procesos que se describen a continuación fue medida utilizando un magnetómetro de giro AGICO JR-6 (Dual Speed Spinner Magnetometer).

La adquisición de MRA es producida por medio de la combinación de un campo magnético estacionario $(\mathrm{H})$ y un campo alterno $(\mathrm{CA})$. El efecto del campo $(\mathrm{H})$ es superpuesto al del campo CA mientras éste decrece lentamente desde su valor máximo hasta cero. En este proceso el campo CA cumple el papel de relajar el sistema y reorientar los momentos magnéticos dipolares permanentes en forma aleatoria. La MRA se indujo siguiendo la técnica de Dunlop and Özdemir (1997) en las muestras, aplicando, en 3 ejes ortogonales, un campo pico de corriente alterna (AC) de $100 \mathrm{mT}$ y uno de corriente directa (CD) de $50 \mu \mathrm{T}$ utilizando un desmagnetizador por campos alternos LDA-3, trabajando en modo de magnetizador.

Sucesivamente, por medio de un magnetizador de pulsos IM-10 (ASC Scientific), se indujo una 
MRI al someter las muestras a una serie de pulsos de $\mathrm{CD}$ que se incrementaron paulatinamente, en etapas de 25, 50, 75, 100, 200, 300, 500 y 700 mT. Una vez alcanzado el campo máximo disponible, se aplicó un campo inverso de $300 \mathrm{mT}$. Previamente a la obtención de la MRI se llevó a cabo una desmagnetización total empleando un campo magnético de $100 \mathrm{mT}$ en el mismo desmagnetizador LDA-3.

A partir de las mediciones anteriores se calcularon los siguientes cocientes inter paramétricos asociados: $\mathrm{S}_{300}, \mathrm{SIRM} / x, \mathrm{ARM} / x$ y ARM/SIRM (Kirschvink, 1980), siendo:

$$
S_{300}=\frac{I R M_{-300}}{I R M_{\text {sat }}}
$$

Considerando la MRI adquirida a $700 \mathrm{mT}$ como la IRM de saturación IRM sat .

\subsection{ANÁLISIS DE GARBONO ORGÁNICO TOTAL (COT)}

Consistió en pesar sub-muestras de $80 \mathrm{mg}$ de los sedimentos molidos en mortero de ágata, de donde se extrajo el carbono a través de la calcinación de la muestra para una posterior titulación del $\mathrm{CO}_{2}$ formado en una solución ácida. La determinación del carbono se llevó a cabo mediante un Coulómetro modelo 5014 de la marca UIC.

\subsection{FECHAMIENTO POR RADIOGARBONO GON AMS}

Directamente en campo, se tomó muestra de materia orgánica con contenido de carbón. Cuatro muestras fueron enviadas a los laboratorios Beta Analytic para ser fechadas a través del método de radiocarbono con AMS, cuyos resultados se muestran la tabla 1. La espectrometría de masas con aceleradores (AMS) consta de dos fases: la primera consiste en la aceleración de los iones a altas energías cinéticas y la segunda es el análisis de masa. Cada muestra es tratada y convertida en un tipo de grafito sólido, convirtiendo dióxido de carbono en presencia de un catalizador metálico.
Posteriormente, las muestras se introducen en un disco de metal junto con otros materiales de referencia. Los materiales se bombardean de Cesio contra la rueda de referencia, con lo que se producen átomos de carbono ionizados negativamente. Luego éstos pasan al stripper (un gas o lámina de metal) para perder electrones y emerger como átomos triples. A partir de aquí, los átomos positivos pasan a través de un conjunto de dispositivos de enfoque para el análisis de masa, en el cual se aplica un campo magnético a las partículas positivas en movimiento, lo que las desvía de su ruta; las partículas más pesadas se desvían con menor intensidad. Los detectores de diferentes ángulos de desviación cuentan las partículas y es posible obtener el número de átomos de carbono 14, carbono 13 y carbono 12 de la muestra, con lo que es posible evaluar la concentración de los isótopos y el nivel de fraccionamiento (Beta Analytic, Testing Laboratory, https://www.radiocarbon.com/espanol/acelerador-masa-espectrometria.htm).

\section{Resultados}

\subsection{GEOGRONOLOGÍA}

El control cronológico del yacimiento, compuesto de una sucesión limo arcillosa en la base y más diatomácea en la cima, se estableció con base en tres fechamientos de sedimentos con valores más altos de TOC a lo largo de la secuencia (Tabla 1), uno en la base y dos en el centro de la sucesión sedimentaria. Los fechamientos fueron realizados en beta analytic mediante AMS C14 y calibrados a años calendáricos con IntCal13 (Reimer et al., 2013). El modelo de edad-profundidad se construyó basándose en la interpolación de las edades adyacentes con un $95.4 \%$ de probabilidad.

Cabe señalar que la accesibilidad a las paredes del afloramiento fue limitada hacia la cima de la secuencia, sin embargo, un ulterior acceso a la mina permitió cubrir el espesor total del yacimiento. Por lo anterior, el nivel 0 es la base de la columna y la cima alcanza los $6.90 \mathrm{~m}$ de espesor. Se obtuvieron tres fechas: la primera a $40 \mathrm{~cm}$ de 
Tabla 1. Fechamientos AMS de la diatomita de Agostitlán.

\begin{tabular}{|c|c|c|c|c|}
\hline $\begin{array}{l}\text { Código } \\
\text { BETA }\end{array}$ & $\begin{array}{l}\text { Clave envio } \\
\text { (profundidad) }\end{array}$ & $\begin{array}{l}\text { Tipo de } \\
\text { muestra }\end{array}$ & $\begin{array}{c}\text { Edad } \\
\text { convencional }\end{array}$ & CALIBRACIÓN sigma 2 \\
\hline 458434 & AGOS $650 \mathrm{~cm}$ & $\begin{array}{l}\text { Sedimentos } \\
\text { lacustres }\end{array}$ & $20,950 \pm 70 \mathrm{BP}$ & $\begin{array}{l}\text { Cal BC } 23510-23200 \\
(\mathrm{Cal} \text { BP } 25460-25150)\end{array}$ \\
\hline 458435 & AGOS $100 \mathrm{~cm}$ & $\begin{array}{l}\text { Sedimentos } \\
\text { lacustres }\end{array}$ & $25,830 \pm 110 \mathrm{BP}$ & $\begin{array}{l}\text { Cal BC } 28455-27735 \\
(\mathrm{Cal} \text { BP } 30405-29685)\end{array}$ \\
\hline 457971 & AGOS $40 \mathrm{~cm}$ & $\begin{array}{l}\text { Sedimentos } \\
\text { lacustres }\end{array}$ & $32,540 \pm 180 \mathrm{BP}$ & $\begin{array}{l}\text { Cal BC } 34840-34160 \\
(\mathrm{Cal} B P 36790 \text { to } 36110)\end{array}$ \\
\hline
\end{tabular}

la base de 36,450 años cal aP., a $1 \mathrm{~m}$ se obtuvo una edad de 30,045 años cal aP. y la cima a 650 cm corresponde a 25,305 años cal aP. Las tres permiten asignar un intervalo de tiempo en el que se llevó a cabo el depósito de la diatomita.

La extrapolación lineal de las cuatro fechas más antiguas indica una edad de $c a$. 40,000 años aP. para la base del registro, mientras que en la cima la secuencia de sedimentación lacustre llega de $c a$. 22.000 años aP. El intervalo de tiempo para que se desarrollara la diatomita se encuentra dentro del Wisconsin tardío (Clayton y Moran,1982) hasta el Último Máximo Glacial. A escala global, corresponde a la parte final del estadio isotópico marino MIS3 (Agosta et al., 2016).

Las tasas de sedimentación se calcularon entre los niveles fechados, considerando que es una sucesión muy homogénea y que los espesores de las cenizas volcánicas son muy pequeños (menos de un $1 \mathrm{~cm}$ ). Las tasas de sedimentación para la secuencia basal pseudolaminada de limos y arcillas de 0 a $100 \mathrm{~cm}$ (ca. 40,000 años aP ? 30,045 años aP) es de $1.6 \mathrm{~mm}$ por año. La sucesión diatomítica más pura de 100 a $650 \mathrm{~cm}(30,045$ a 25,305 años aP) presenta una tasa de sedimentación mucho más baja de $0.093 \mathrm{~mm} /$ año.

\subsection{ESTRATIGRAFÍA}

La columna sedimentaria agrupa 3 unidades estratigráficas principales (Figura 3). La unidad I, que corresponde a la base de la secuencia, tiene un espesor de $180 \mathrm{~cm}$. Consiste en una alternancia de limos intercalados con arcillas finas con espesores de 1 a $3 \mathrm{~cm}$. En general los sedimentos son pseudolaminados y de color más oscuro en compara- ción con los sedimentos del resto de la sucesión. Un depósito de ceniza de $3 \mathrm{~cm}$ se distingue de 70 a $73 \mathrm{~cm}$. Hacia la cima la sucesión se hace más detrítica fina limosa, presentando deformación sinsedimentaria y microfallas. Culmina dicha unidad con un depósito de $1 \mathrm{~cm}$ de tefra. La unidad II se encuentra por encima de una tefra color gris oscuro y tiene un espesor de $320 \mathrm{~cm}$. En su base se observa un color obscuro de diatomita arcillosa beige con abundantes bioturbaciones a techo de los estratos basales que, transicionalmente, pasan a una diatomita masiva color blanco que presenta dos horizontes laminados con pequeños niveles oxidados a 200 y $230 \mathrm{~cm}$. En este segundo paquete se intercalan dos estratos de cenizas finas con espesores menores a $1 \mathrm{~cm}$ color gris oscuro a negro (235 y $360 \mathrm{~cm}$ ). En esta unidad se encuentran abundantes escamas de peces y huellas de bioturbación, así como restos de posibles moluscos. La unidad III se encuentra en contacto transicional con la anterior y corresponde a una diatomita de color blanco con textura un poco limosa y más deleznable, la cual presenta un nivel oxidado a $430 \mathrm{~cm}$ de $0.8 \mathrm{~cm}$ de espesor y una tefra fina de gris claro a $540 \mathrm{~cm}$ y en donde se encuentran abundantes escamas de peces y restos de raíces.

\subsection{ANÁLISIS DE DIATOMEAS}

Se analizó el registro taxonómico de la diatomita de Agostitlán, obteniéndose un listado de 31 especies con predominancia de formas Centrales planctónicas, siendo características y dominantes Aulacoseira spp. y Stephanodiscus spp. La flora ticoplanctónica incluye formas de la familia Fragilariaceae (Williams y Round 1987) y, en escasos porcentajes, algunas especies perifiticas (Kociolek et al., 2014) que incluyen a Cocconeis spp., Navicula spp., Gomphonema spp., Epithemia spp. y Cymbella spp. y algunos géneros de preferencia bentónica como Nitzchia spp., Sellaphora spp, Stauroneis spp. La diatomita presenta una abundancia de hasta $1.8 \times 10^{7}$ valvas/g de sedimentos secos.

El registro de sedimentación biógena a lo largo de la columna estratigráfica del yacimiento se realizó por zonas, de acuerdo a los cambios 


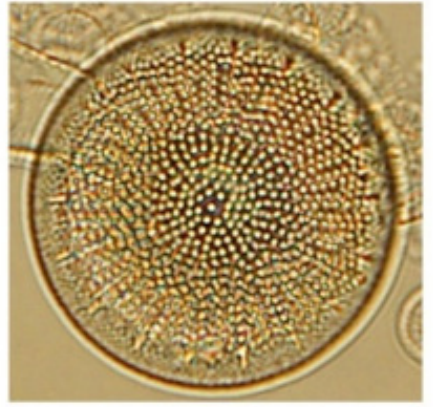

1 a

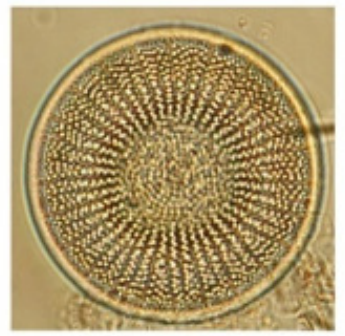

2

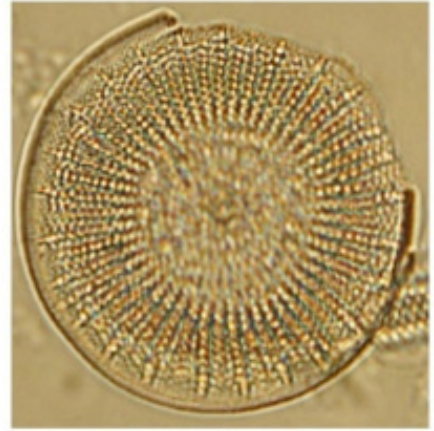

1b

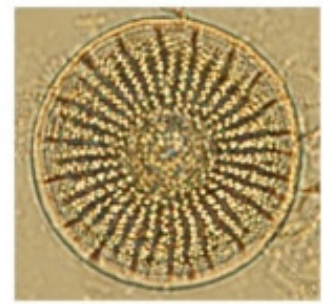

3

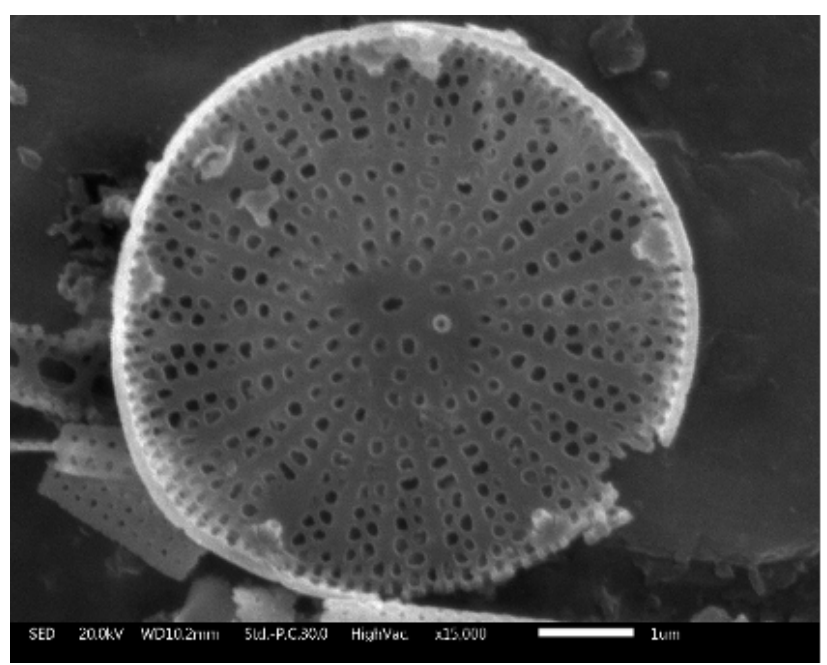

$4 d$

Figura 4 1a-1b) Stephanodiscus sp. aff Stephanodiscus niagarae (d=25 $\mu \mathrm{m}, 28 \mu \mathrm{m} ; 6$ areolas/10 $\mu \mathrm{m})$. Vista valvar. 2) Stephanodiscus sp. aff Stephanodiscus transilvanicus $(\mathrm{d}=19 \mu \mathrm{m})$. Vista valvar.3) Stephanodiscus sp. aff Stephanodiscus carconensis $(\mathrm{d}=18 \mu \mathrm{m})$. Vista valvar. 4a-4c) Stephanodiscus minutulus ( $\mathrm{d}=6 \mu \mathrm{m}, 6 \mu \mathrm{m} ; 7 \mu \mathrm{m}$ ). Vista valvar. 4d)- Imagen obtenida con un microscopio electrónico de barrido (MEB). Vista interna de la valva de Stephanodiscus aff. minutulus con hileras de aureolas irregulares uni a biseriadas. Al interior de las aureolas se observa la criba enfosada. Se distingue un proceso reforzado desplazado a la derecha en el centro de la valva. En los márgenes se observan cinco procesos reforzados con dos poros satélite cada una de ellos.

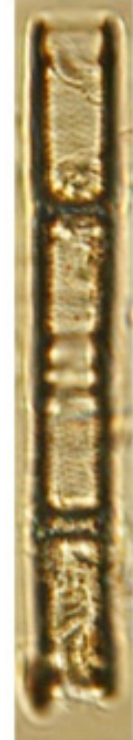

1
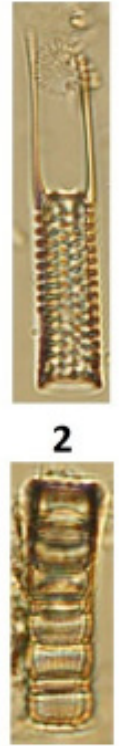

6a

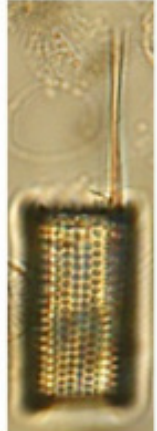

3

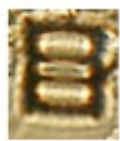

6b

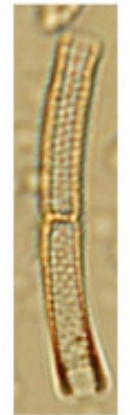

4

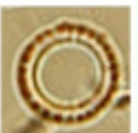

$7 a$

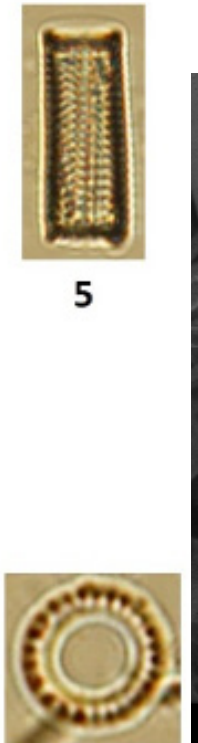

7b

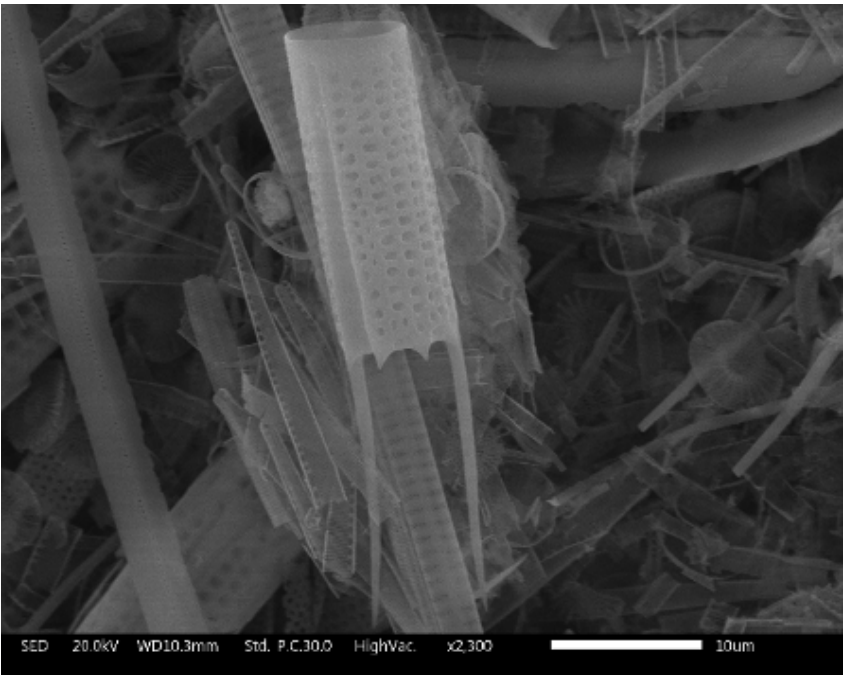

$7 \mathrm{c}$

Figura 5 1) Aulacoseira sp. aff. Aulacoseira italica ( $\mathrm{L}=14 \mu \mathrm{m}-\mathrm{A}=4 \mu \mathrm{m})$. Vista de manto. 2) Aulacoseira granulata var. angustissima $(\mathrm{L}=11 \mu \mathrm{m}-\mathrm{A}=3 \mu \mathrm{m})$. Vista de manto SEM. 3) Aulacoseira granulata var. monospina $(\mathrm{L}=10 \mu \mathrm{m}-\mathrm{A}=6 \mu \mathrm{m})$. Vista de manto. 4) Aulacoseira granulata var. angustissima $(\mathrm{L}=10 \mu \mathrm{m}-\mathrm{A}=2 \mu \mathrm{m})$. Vista de manto. 5) Aulacoseira granulata $(\mathrm{L}=11 \mu \mathrm{m}-\mathrm{A}=4 \mu \mathrm{m})$. Vista de manto. $6 \mathrm{a}-6 \mathrm{~b})$ Aulacoseira spp $(\mathrm{d}=5 \mu \mathrm{m})$. Vista de manto.7a-7b) Aulacoseira sp. aff Aulacoseira granulata $(\mathrm{d}=6 \mu \mathrm{m})$. Vista valvar. 7c) Aulacoseira granulata var. angustissima (Imagen MEB). 


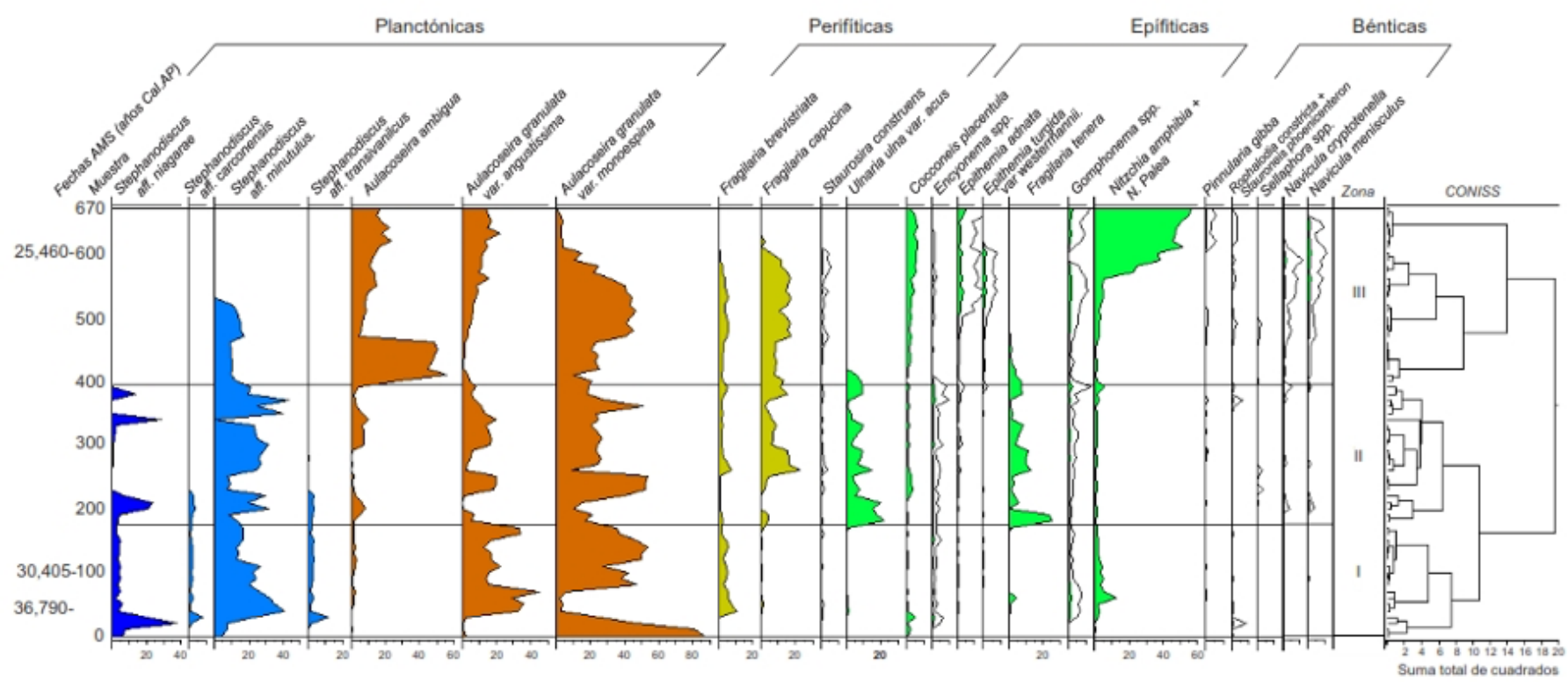

Figura 6 Registro de diatomeas del paleolago de Agostitlán, ordenado según CONISS en tres zonas donde se observa el dominio de las formas planctónicas entre ca. 40 y 25 ky. Posterior a ca.25ky se observa una regresión del lago con aumento de formas epifiticas.

observados en la sucesión estratigráfica, las cuales corresponden con el ordenamiento que realiza el programa CONISS (Figura 6).

\subsubsection{EPISODIO I (BASE O A 70 CM)}

Durante el primer ciclo de sedimentación de $c a$. 40,000 años cal aP a ca. 34,500 años cal. aP, se registra una abundancia de especies planctónicas, siendo la forma dominante Stephanodiscus minutulus, seguida por $S$. niagarae, S. transilvanicus y $S$. carconensis. En este episodio Stephanodiscus alterna con Aulacoseira, esta última alcanzando dominancias hasta del $90 \%$.

\subsubsection{EPISODIO II (75 A 400CM)}

Este episodio tiene una duración de aproximadamente 6,000 años; a partir de $75 \mathrm{~cm}$ y hasta los $180 \mathrm{~cm}$ (ca. 30,500 años cal aP hasta ca. 28,000 años cal aP) se registra la mayor abundancia de diatomeas, con una productividad algal total de $3.956 \times 10^{9}$ diatomeas por gramo de sedimento. Descienden los porcentajes de Stephanodiscus hasta un $30 \%$ y vienen reemplazados por el aumento de Aulacoseira granulata, A. angustissima y A. monospina, acompañadas de Fragilaria brevistriata $(10 \%)$ y otras formas epifiticas como Encyonema y Navicula en porcentajes menores al $10 \%$.
De 180 a 400 cm, ca. 29,700 años a.P a ca 28,000 años a.P, A. granulata var. angustissima, alternando con Stephanodiscus niagarae, S. aff carconensis y S. minutulus, caracterizan este intervalo. Resalta el inicio y constancia de Ulnaria ulna var. acus, Posteriormente, este episodio II, de 230 a $400 \mathrm{~cm}$, que sigue dominado por la asociación Stephanodiscus-Aulacoseira granulata, se registra aparición y constancia (con un porcentaje mayor a 30\%) del grupo de formas alargadas tendencialmente planctónicas, de Fragilariacea (Ulnaria ulna var. acus, Fragilaria tenera y F. capucina). Al final de este episodio, de 360 a 400 $\mathrm{cm}$, Stephanodiscus aff niagarae y $S$. aff minutulus alternan en abundancia con Aulacoseira granulata var. angustissima y $A$. granulata var. monospina, caracterizando así este episodio, acompañadas de Fragilaria brevistriata, F. capucina, F. tenera y Ulnaria ulna var. acus.

\subsubsection{EPISODIO III (400CM A 668 CM)}

Este intervalo tiene una duración de ca. 3000 años; de ca. 28,000 años cal aP a ca. 25,000 años cal aP se divide en tres fases lacustres, la primera comprende de 400 a $470 \mathrm{~cm}(28,000 \mathrm{aP}$ a 27,750 aP), mostrando un aumento considerable de Aulacoseira ambigua y A. granulata var. monospina y alcanzando hasta el $60 \%$ de dominancia, en 
correspondencia con un descenso de Stephanodiscus minutulus. Resalta la desaparición de Ulnaria ulna y Fragilaria tenera. Diatomeas perifiticas que incluyen Cocconeis placentula var. placentula, Cocconeis placentula var. lineata, Navicula cryptotenella, Navicula menisculus, Gomphonema parvulum, Epithemia adnata, y E. turgida var. westermanni, aumentan paulatinamente.

La segunda va de 470 a $530 \mathrm{~cm}$. (ca. 27,750 años cal aP a 26,500 años cal aP). Este intervalo Aulacoseira granulata var. monoespina de nuevo domina en el registro con más de $60 \%$, es en los últimos niveles donde se registra Stephanodiscus minutulus, cuyos porcentajes descienden hasta alcanzar solo el 20\% del total de las valvas, mientras que especies perifíticas y bentónicas empiezan a aumentar en la columna estratigráfica e incluyen a Epithemia adnata, E. turgida var. westermannii, Nitzchia amphibia. Las concentraciones de TOG permanecen fluctuantes, al igual que los niveles de susceptibilidad magnética. Se presentan dos aumentos significativos en la susceptibilidad magnética, a 490 y 540 $\mathrm{cm}$.
Hacia la cima, durante la última fase ca. 26,500 aP a 25,000 aP (530 a $680 \mathrm{~cm})$, se establece el incremento paulatino de Aulacoseira granulata y $A$. ambigua, adquiriendo importancia Nitzschia amphibia y $\mathcal{N}$. palea, que alcanzan hasta el $60 \%$ de la dominancia en esta zona. Otras especies litorales características, aunque en bajos porcentajes, son Pinnularia y Rhopalodia, además de las ya descritas en la fase anterior con un ligero aumento en su abundancia. Hacia la cima, a $680 \mathrm{~cm}$, se observa un contacto discordante con posibles paleosuelos.

\subsection{PARÁMETROS MAGNÉTICOS}

Los sedimentos lacustres presentan una respuesta magnética relativamente débil debido a la baja concentración de minerales magnéticos. No obstante, la sensibilidad de los equipos utilizados (Kappabridge y magnetómetro de giro JR6) permitieron graficar las variaciones de susceptibilidad magnética y magnetización de saturación (SIRM) vs altura (Figura 7).
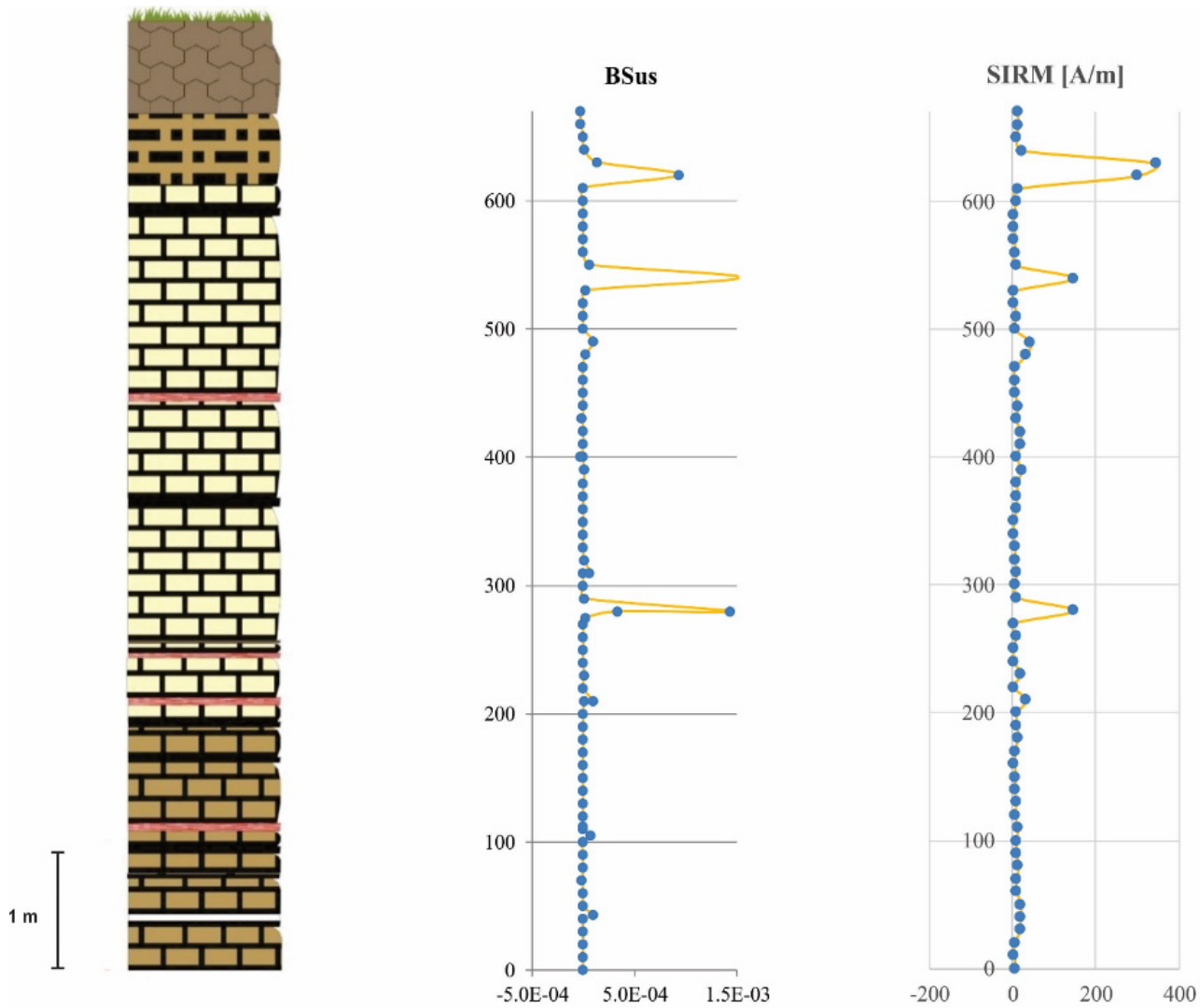

Figura 7 Columna estratigráfica y parámetros dependientes de la concentración ( $\chi$, SIRM). Ambos parámetros magnéticos muestran prácticamente las mismas variaciones con la profundidad a partir de los $200 \mathrm{~cm}$. 
En los registros de susceptibilidad magnética contra la altura (Figura 8) se observan al menos cuatro máximos (picos) a 50, 110, 280 y 320 $\mathrm{cm}$, los cuales se relacionan directamente con máximos de intensidad y porcentaje de carbono $(\% \mathrm{C})$. En el mismo registro también se observan otros dos máximos en susceptibilidad magnética e intensidad que presentan, sin embargo, una relación inversa con mínimos de \% $\mathrm{C}$ a 540 y $620 \mathrm{~cm}$.

La comparación de las gráficas de ARM vs altura y del parámetro $S_{300}$ vs altura contra la abundancia de diatomeas (Figura 9) permite la identificación de dos intervalos característicos de la secuencia sedimentaria: el primero, de 60 $\mathrm{cm}$ a $400 \mathrm{~cm}$, en el cual tanto la ARM, cuanto el cociente $\mathrm{S}_{300}$, se mantienen casi constantes, mientras que la abundancia de diatomeas presenta una disminución significativa del 66\%, al pasar de 6000 a 2000 frústulas. Por el contrario, en el segundo intervalo $(420 \mathrm{~cm}$ a $670 \mathrm{~cm})$ la variabilidad se presenta en ambos parámetros magnéticos $\left(\mathrm{ARM}\right.$ y $\left.\mathrm{S}_{300}\right)$ mientras que la abundancia muestra una disminución progresiva.

La Magnetización Remanente Isotermal (IRM, por sus siglas en inglés) depende de la concentración de minerales ferrimagnéticos. La forma de las curvas de adquisición de esta remanencia es útil para inferir la presencia de minerales de alta coercitividad (hematita y/o goetita) o de minerales multidominio. En la Figura 10 puede observarse cómo la muestra se satura en campos cercanos a 300 mT. En algunos casos lo hace a partir de los $200 \mathrm{mT}$, alcanzando valores cercanos al $100 \%$, lo que indica que se tiene la presencia principalmente de minerales magnéticamente blandos (ferrimagnéticos), muy probablemente titanomagnetita. En general, las gráficas son muy similares en cuanto a la forma para todas las muestras y no hay diferencias contrastantes apreciables.

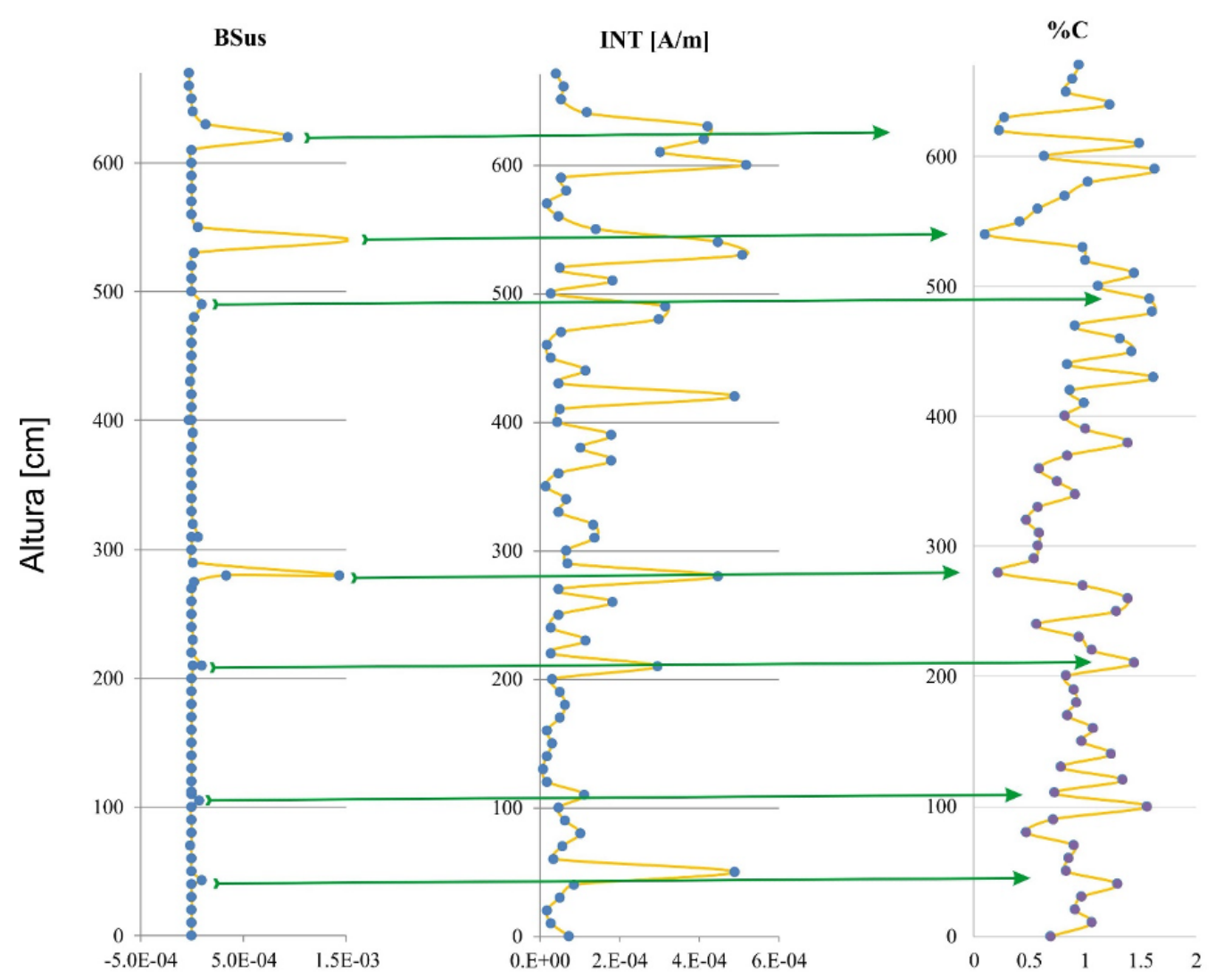

Figura 8 Gráfica de susceptibilidad magnética (Bsus), Intensidad de magnetización (INT) y porcentaje de carbono (\%C) contra profundidad. 


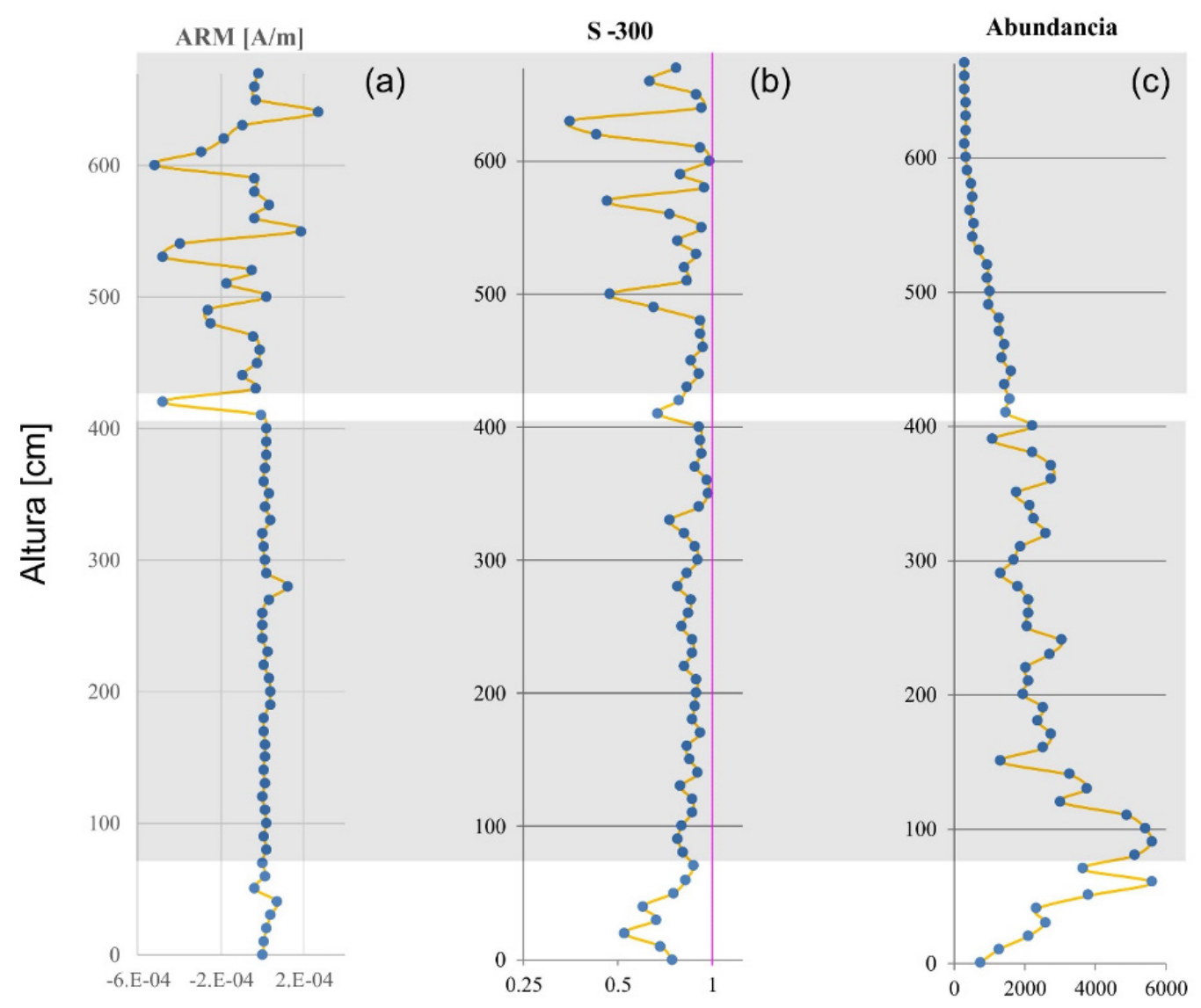

Figura 9 Variación con la altura de (a) intensidad de ARM, (b) cociente S300 y (c) Abundancias de diatomeas. A partir de las gráficas mostradas se pueden identificar dos intervalos característicos de la secuencia sedimentaria. El primero de 0 a $400 \mathrm{~cm}$ con un comprotamiento homogéneo tanto en la ARM y S300. El segundo hacia la cima, de 400 hasta $650 \mathrm{~cm}$, mostrando marcadas fluctuaciones y con un claro descenso en la abundancia de diatomeas.

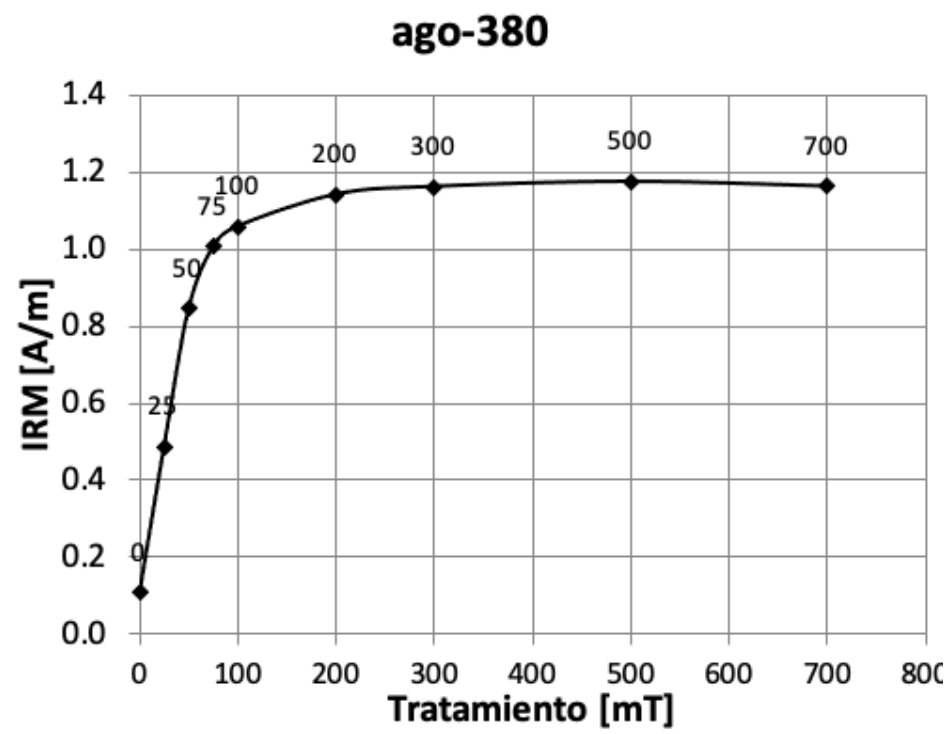

Figura 10 Curva de adquisición de Magnetización Remanente Isotermal. Se observa cómo la muestra satura a partir de los 200 mT. 


\section{Discusión}

Al final del interglacial $c a$. 40,000 años aP y hasta $c a$. 34,500 años aP, al final del MIS3 en el denominado pre Wisconsin tardío, se estableció una cuenca por el represamiento del río Tuxpan, por la colada de lava del estratovolcán Cerro de la Soledad, al SO de la cabecera municipal de Tuxpan y en donde el drenaje fluvial principal fue represado rápidamente. Tres paquetes sedimentarios son notorios por sus cambios de coloración y textura y se reflejan también en cambios en la composición de las agrupaciones de diatomeas.

En la sucesión lacustre que enmarca la zona 1 Aulacoseira granulata domina base. Esta especie es considerada una diatomea termófila asociada a temperaturas mayores a $\operatorname{los} 15^{\circ} \mathrm{C}$ (Rioual et al., 2007). Hacia la cima viene substituida por la asociación Stephanodiscus niagarae- Stephanodiscus aff.medius y Aulacoseira granulata que, en conjunto, caracterizan ambientes con tendencia a profundos. Esta asociación, ascendiendo estratigráficamente, viene acompañada por las formas betónicas Nitzschia amphibia- Nitzschia y N. palea (Kociolek et al., 2014), sugiriendo un descenso del nivel del lago y un incremento de la turbidez, corto periodo (incremento en la susceptibilidad magnética). No obstante, de nuevo aumentan especies indicadoras de hábitats planctónicos; aunque se mantiene bajo, no se alcanzan las profundidades iniciales del lago. En general, las especies de Stephanodiscus (Figura 4), con la posible excepción de Stephanodiscus niagarae, florecen en la primavera durante condiciones de niveles bajos pero crecientes de luz y nutrientes, particularmente fósforo (Bradbury, 1988). En el Oeste de Estados Unidos, Stephanodiscus niagarae actualmente florece en el otoño, lo que implicaría temperaturas más bajas que las actuales.

En este intervalo se alcanzan las máximas abundancias de diatomeas en el registro sedimentario, llegando a presentar hasta $5 \times 10^{9}$ valvas por gramo de sedimento. La sílice lixiviada del entorno probablemente facilitó que se alcanzaran estas abundancias.

Hacia la zona II (180 a $400 \mathrm{~cm}$ ) se observa la reaparición de $S$. niagarae y $\mathrm{S}$. aff. medius. El inicio y el final de dicha zona sugiere que el lago se profundiza de nuevo, presentando condiciones lacustres con mayor cantidad de nutrientes. Destaca la aparición y constancia de formas alargadas del grupo Fragilaria solo en esta zona. Estas especies largas y delgadas viven en suspensión en la columna de agua y comúnmente se observan asociadas a Stephanodiscus (Davies et al, 2002).

Se presenta un pico, un aumento significativo en la susceptibilidad magnética a la mitad de este episodio lacustre que se asocia a incremento de la turbidez en el contexto de un clima húmedo (Figura 7).

En el inicio de la tercera fase del lago (hacia el final del último interglacial) se presentó un aumento significativo de Aulacoseira ambigua, que se convierte en la especie dominante de 400 a 460 cm, ca. 26,000 a 27,000 años aP). A. ambigua se ha encontrado en registros Holocénicos del centro de México, principalmente en la meseta tarasca, Zacapu (Metcalfe, 1995), Zirahuén (Davies et al., 2004) y Pátzcuaro (Bradbury 2000). Se considera que es una especie que vive en sitios con abundante luz, floreciendo durante la primavera y el verano en ambientes tendientes a fríos con alto contenido en nutrientes (Wang et al., 2008).

La presencia de Aulacoseira ambigua en Pátzcuaro, entre ca. 38,000 y 34,000 años aP, sugiere climas fríos (Bradbury, 2000). Al parecer esta tendencia a condiciones frías continúa hacia 26,000 años dada la presencia de bosques de Abies observados en sedimentos fluvio lacustres en el margen oeste del Lago de Pátzcuaro (Robles-Camacho et al., 2010). En el margen sur del mismo las diatomitas levantadas por efecto de la construcción del volcán la Tasa consisten de asociaciones de Stephanodiscus niagarae, S minutulus y Aulacoseira granulata y Aulacoseira ambigua fechadas en 32,776 años cal bP (Israde et al., 2005), sugiriendo así condiciones tendientes a frías.

A partir de 25,000 años cal aP y hacia el inicio del Último Máximo Glacial 22,000 cal aP (Lambeck et al, 2014, Clark et al., 2009), la cima del registro está caracterizada por dominancia Nitzschia amphibia y Nitzschia palea en substitución de Aulacoserira ambigua, que desciende hasta menos 
del 20\% de su abundancia. Y otras diatomeas bentónicas (Epithemia, Gomphonema, Cocconeis) indican un descenso del nivel lacustre y la instalación de un cinturón de macrofitas.

Durante el desarrollo del lago fue fluctuante y turbio, pero profundo con un cambio a bajos niveles lacustres al final del MIS3. Este cambio puede estar asociado a la apertura de drenaje hacia el principal afluente, el río Tuxpan.

La susceptibilidad magnética es la respuesta de un material a la aplicación de un campo magnético de baja intensidad (Butler, 1998) y nos permite reconocer la variabilidad de las concentraciones de minerales magnéticos. También puede ser un indicador para determinar periodos con condiciones ambientales estables, debido a que los periodos de mayor erosión y arrastre superficial de sedimentos frecuentemente están asociados a decrementos en la cobertura vegetal y/o a incrementos en la precipitación.

Los parámetros dependientes de la concentración $(x, \mathrm{SIRM})$ muestran las mismas variaciones con la profundidad. Los valores más altos de $x$ (Figura 7) se presentan a 50, 110, 210, 280, 320, 490,540 y $620 \mathrm{~cm}$ ), en coincidencia con niveles más oscuros en el depósito.

La correlación negativa entre $x$ y TOC podría indicar que para esos depósitos las variaciones de susceptibilidad se deben a la dilución de minerales magnéticos por materia orgánica, no magnética. Sin embargo, esta correlación inversa no está clara para todas las unidades estratigráficas y sugiere que otros factores como la disolución o la autogénesis pueden haber afectado estos sedimentos. La correlación positiva entre $x$ y SIRM (Figura. 7) muestra que ambos dependen, principalmente, de la concentración de minerales magnéticos, pero también se sugieren otras causas como la mineralogía, el tamaño de grano y el contenido paramagnético.

El parámetro $\mathrm{S}_{300}$ y la abundancia de diatomeas permanece fluctuante con máximos y mínimos (Figura 9) que algunas veces se corresponden directamente $(50,60,150,170,210,240,250,330$, $410 \mathrm{~cm})$ y algunas otras de manera inversa $(70,90$, $110,130,350,390)$ hasta $400 \mathrm{~cm}$ de profundidad, mientras que la intensidad ARM permanece prác- ticamente constante de manera lineal y a partir de $410 \mathrm{~cm}$. El cociente $\mathrm{S}_{300} \mathrm{y}$ la intensidad ARM se vuelven muy fluctuantes con máximos y mínimos muy marcados, coincidiendo con la caída en la abundancia relativa de diatomeas y, por tanto, en la productividad algal.

El uso de los cocientes magnéticos, dependientes del tamaño de partícula ARM/SIRM y ARM/ $x$, permiten mostrar la contribución de partículas magnéticas finas y gruesas. Por lo tanto, valores más altos de estos parámetros indicarían la presencia de granos ultrafinos ( $\sim 0.1 \mu \mathrm{m}$ o menores). Dichos parámetros exhiben un comportamiento muy similar a la susceptibilidad magnética. La relación inversa entre $x$ y ARM/SIRM indica que los sedimentos de menor concentración también tienen los granos más pequeños, aunque las tefras forman su propio grupo de alta concentración y de grano grande.

El cociente SIRM/ $x$ se relaciona con la mineralogía magnética; valores más altos de estos parámetros indican una coercitividad más grande.

Como la composición de las titanomagnetitas es diversa en todos los niveles de cenizas y sedimentos lacustres, consideramos que las características magnéticas observadas en los sedimentos lacustres, donde los valores bajos de $\mathrm{S}_{-300}$ coinciden con los valores bajos de ARM / SIRM, reflejan, principalmente, cambios en las mezclas minerales de titanomagnetitas y goetitas o hematitas, en lugar de la mezcla de diferentes tamaños de titano magnetitas de la misma composición. Esta mezcla mineral resulta de la erosión de los materiales volcaniclásticos y de los productos del suelo.

\section{Variabilidad climática en el centro de México para el Pleistoceno tardío y su relación con los forzamientos a escala global}

En el final del último ciclo glacial, que abarca entre 60,000 y 27,000 años y corresponde al estadío isotópico MIS 3, se desarrolló el paleolago de Agostitlán entre ca. 26,000 y 40,000 años cal aP. Durante este corto intervalo de alta variabilidad climática a nivel global se presentaron condiciones 
interestadiales que se enmarcan dentro de dos eventos más fríos (MIS 4 y MIS 2). Las temperaturas interestadiales pudieron subir en pocas décadas (Van Meerbeeck et al., 2009) y, en conjunto con un aumento del nivel del mar, produjeron un aumento de humedad y de precipitación por efecto de mayor actividad monzónica, lo que afectó los trópicos (Deplazes et al., 2018). El registro de Agostitlán presenta tres episodios de alto nivel lacustre a ca. 27,000 a 29,000 años aP, y a ca. 40,000 años aP., que pueden compararse con los registros climáticos globales, los eventos de mayor humedad y aumento de temperatura (Heinrich $\mathrm{H} 2, \mathrm{H} 3$ y H4, respectivamente).

Posterior a los ca. 26,000 y 27,000, la aparición de Aulacoseira ambigua sugiere temperaturas más bajas que las actuales.

En Pátzcuaro (localizado ciento veinte kilómetros al Oeste de Agostitlán, a una altitud de 2095 metros sobre el nivel del mar), condiciones más frías se presentan previamente a ca. 37,000 dada la presencia de Aulacoseira ambigua en el lago, mientras que en los depósitos fluviolacustres estas condiciones frías se confirman por la presencia de polen de Abies hacia los 31,600 años cal aP (Robles Camacho et al., 2010). Condiciones húmedas también se observan en la cuenca de Cuitzeo hacia 40,000 años cal aP (Israde et al., 2010b) y Zacapu antes de 35,000 años aP, volviéndose más secas posteriormente (Ortega et al., 2002).

La configuración orográfica del entorno del paleolago de Agostitlán con elevaciones de ca. 2200 metros pudieron favorecer condiciones frías para el desarrollo de Aulacoseira ambigua, cuya máxima abundancia alcanza entre 26,000 y 27,000 años aP.

Se ha sugerido que los registros al oeste de la Cuenca de México indican mayor humedad durante el periodo Glacial y Postglacial con una tendencia espacial decreciente de la humedad del occidente al oriente en el centro de México (Bradbury, 1997). Agostitlán era predominantemente profundo y de agua dulce de 40,000 a ca.30,000 años aP. En consecuencia, la humedad del oeste pudo haber desempeñado un papel esta- cional, especialmente en el centro oeste de México en ese momento. Consideramos que se requieren más registros que cuenten con varios proxies para entender la señal climática de este intervalo en México.

A pesar de que este lago se formó por un represamiento rápido con el sucesivo desarrollo de taxa de hábitats planctónicos, es consistente con el desarrollo de lagos con cierta estabilidad, siguiendo una tendencia a altos niveles lacustres hasta el final del Interglacial en varios sectores de México, incluidos Chalco (Caballero y Ortega, 1998) y el norte de México (Roy et al., 2014). Posterior a ca. 28,000 años aP., los lagos del centro de México, incluido Agostitlán, mostraron una tendencia a somerizarse (Pátzcuaro: Bradbury, 2000; Zacapu: Metcalfe, 1992: Ortega et al., 2002).

\section{Conclusiones}

Los resultados de análisis de diatomeas y de magnetismo reconstruyen la historia paleoambiental de Agostitlán durante los últimos 40,000 años aP, permitiendo con ello contribuir a la comprensión de los cambios climáticos durante la transición Pleistoceno-Holoceno en esta porción del Centro Oeste de México.

El sistema lacustre fue alimentado por los drenajes provenientes de las sierras de Agostitlán, siendo la sílice, para el desarrollo de la diatomita, proporcionada por los numerosos aparatos volcánicos que lo rodean.

Se observa que la diatomita se desarrolló en tres fases: Durante la primera, hace $c a$. 40,000 años aP, se establece un cuerpo de agua con nivel lacustre estable con tendencia a cierta turbidez, con alternancia de eventos volcánicos con diatomitas de alta pureza, que pasa sucesivamente durante una segunda fase a un cuerpo de agua relativamente profundo y poco turbio. Es en ésta donde la columna de agua alcanza su mayor productividad algal, de 28,000 a 25,000 años aP. En la cuenca vecina, al sureste de Pátzcuaro, durante el Último Máximo Glacial, se establecen condiciones húme- 
das con altos niveles lacustres y, probablemente, las facies profundas observadas en la cuenca de Zacapu puedan corresponder a esta misma fase o periodo, donde se conforman los mayores espesores de la secuencia sedimentaria. Posteriormente, en la tercera fase se registra una serie de fluctuaciones en el nivel lacustre, que culminan con un descenso muy significativo, con ambientes turbios con aporte de materia orgánica ca. 18,000 años cal aP. al final de la deglaciación.

A pesar de que este lago se formó por un represamiento rápido, con el sucesivo desarrollo de taxa de hábitats planctónicos, el final del Interglacial en varios sectores de México es consistente con el desarrollo de ambientes lacustres altos.

Agostitlán fue predominantemente profundo y de agua dulce de 40-30,000 años cal aP., siendo su fuente de aporte los aportes de humedad del oeste, especialmente en el centro oeste de México. Posterior a los 25,000 aP desciende el nivel del lago hasta convertirse en una Ciénega.

\section{Agradeciminetos}

Los autores dan gracias a los revisores anónimos del manuscrito por sus sugerencias. Isabel Israde agradece a su esposo Víctor Hugo Garduño Monroy por su impulso para dar a conocer la evolución paleoambiental de la zona. A la Coordinación de Investigación Científica de la Universidad Michoacana por su apoyo al proyecto CIC 2019. A Salvador Vázquez, asistente editorial del BSGM.

\section{Referencias}

Agosta, A.E., Compagnucci, R.,2016,Abrupt climate changes during the marine isotope stage 3 (MIS 3), en Gasparini G., Rabassa J., Deschamps C., Tonni E. (eds), Marine Isotope Stage 3 in Southern South America, 60 KA B.P.-30 KA B.P., Springer Earth System
Sciences: New York, Springer, 81-106.https:// doi.org/10.1007/978-3-319-40000-6_5

Aranda-Gómez, J.J., Luhr, J.F., Housh, T.B, Valdez-Moreno, G., Chávez-Cabello, G., 2005, El volcanismo tipo intraplaca del Cenozoico tardío en el centro y norte de México: una revisión: Boletín de la Sociedad Geológica Mexicana, 57, 187-225.https:// doi.org/10.18268/bsgm2005v57n3a1

Bidegain, J.C., Rico, Y., Bartel, A., Chaparro, M.A.E., Jurado, S., 2009, Magnetic parameters reflecting pedogenesis in Pleistocene loess deposits of Argentina: Quaternary International, 209(1-2), 175-186.https://doi.org/10.1016/j. quaint.2009.06.024

Bradbury, J.P., 1988, A climatic-limnologic model of diatom succession for paleolimnological interpretation of varved sediments at Elk Lake, Minnesota: Journal of Paleolimnology, 1, 115-131. https://doi.org/10.1007/ bf00196068

Bradbury, J.P., 1997, Sources of glacial in Mesoamerica: Quaternary International, 43-44, 97-110. https://doi.org/10.1016/ s1040-6182(97)00025-6

Bradbury, J.P., 2000, Limnologic history of Lago de Pátzcuaro, Michoacán, México for the past 48,000 years: impacts of climate and man: Palaeogeography, Palaeoclimatology, Palaeoecology, 163, 69-95. https://doi. org/10.1016/S0031-0182(00)00146-2

Bradbury, J.P., Krebs W.N., 1982, Neogene and Quaternary lacustrine diatoms of the western Snake River basin, Idaho-Oregon, USA: Acta Geologica Acaderniae Scientiarum Hungaricae, 25 (1-2), 97-122. https://doi. org/10.2307/3514621

Butler, R.F., 1998, Paleomagnetism: magnetic domains to geologic terranes, Edición electrónica: Departamento de Geociencias de la Universidad de Arizona, USA.

Caballero, M., Ortega, B., Valadez, F., Metcalfe, S., Macias, J.L., Sugiura, Y., 2002, Santa Cruz Atizapán: a 22-ka lake level record and 
climatic implications for the late Holocene human occupation in the upper Lerma Basin, Central Mexico: Palaeogeography, Palaeoclimatology, Palaeoecology, 186, 217-235. https://doi.org/10.1016/ S0031-0182(02)00502-3

Caballero, M., Ortega, B., 1998, Lake levels since about 40,000 years ago at Lake Chalco, near Mexico City: Quaternary Research, 50, 69-79. https://doi.org/10.1006/ qres.1998.1969

Chaparro, M.A.E., 2006, Estudio de Parámetros Magnéticos de Distintos Ambientes Relativamente Contaminados en Argentina y Antártida, Monografías del Instituto de Geofísica, Universidad Nacional Autónoma de México, Grupo de Geomagnetismo, IFAS, Universidad Nacional del Centro de la Provincia de Buenos Aires (UNCPBA).

Clark, P.U., Dyke, A.S., Shakun, J.D., Carlson, A.E., Clark, J., Wohlfarth, B., Mitrovica, J.X.,Hostetler, S.W., McCabe, A.M., 2009, The Last Glacial Maximum: Science, 325, 710-714. https://doi.org/10.1126/ science. 1172873

Clayton, L., Moran, R.S., 1982, Chronology of late Wisconsinan glaciation in middle North America: Quaternary Science Reviews, 1(1), 1982, 55-82. https://doi. org/10.1016/0277-3791(82)90019-1

Davies S., Metcalfe, S., Caballero, M., Juggins, S., 2002, Developing diatom-based transfer functions for Central Mexican lakes: Hydrobiology, 467, 1-3, 199-213. https:// doi.org/10.1007/978-94-010-0415-2_18

Davies S.J., Metcalfe S.E., MacKenzie A.B., Newton A.J., Endfield G.H., Farmer J.G., 2004, Environmental changes in the Zirahuen Basin, Michoacan, Mexico, during the last 1000 years: Journal of Paleolimnology, 31, 77-98. https://doi. org/10.1023/B:JOPL.0000013284.21726.3d

Deino A.L., Kingston J. D., Glen J.M., Edgar R.K., Hill A., 2006, Precessional forcing of lacustrine sedimentation in the late Cenozoic Chemeron Basin, Central Kenia
Rift, and calibration of the Gauss/Matuyama boundary: Earth and Planetary Science Letters, 247(1-2), 41-60. https://doi.org/10.1016/j. epsl.2006.04.009

Deplazes, G., Meckler, A.N., Peterson, L.G., Hamann, Y., Aeschlimann, B., Günther, D., Martínez-García, A., Haug, G.H., 2018, Fingerprint of tropical climate variability and sea level in sediments of the Cariaco Basin during the last glacial period: Journal of Sedimentology, 66(5),1967-1988. https://doi. org/10.1111/sed.12567

Dunlop, D.J., Özdemir, Ö., 1997, Fundamentals of magnetism, en Rock Magnetism: Fundamentals and Frontiers: Cambridge, Cambridge University Press, 16-44. https:// doi.org/10.1017/CBO9780511612794.003

Fisher, C.T., Pollard, H.P., Israde, I., Garduño, V.H., Banerjee, S., 2003, A reexamination of human induced environmental change within the Lake Pátzcuaro Basin, Michoacán, Mexico: Proceedings of the National Academy of Sciences, 100 (8), 4957-4962. https://doi. org/10.1073/pnas.0630493100

Fourtanier, E., Gasse, F., 1988, Premiers jalons d'une biostratigraphie et evolution des diatomées lacustres d'Afrique depuis 11 Ma: Comptes Rendus de l'Académie des Sciences de Paris, 306(2),1401-1408.

Fourtanier, E., Gasse, F., Bellier, O., Bonhomme, M., Robles, I., 1993, Miocene non marine diatoms from western cordillera basins of Northern Peru: Diatom Research, 8(1), 13-30. https:// doi.org/10.1080/0269249X.1993.9705236

Garduño-Monroy V.H., Gutiérrez-Negrín L.C.A, 1992, Magmatismo, hiatus y tectonismo de la Sierra Madre Occidental y del Cinturón Volcánico Mexicano: Geofísica Internacional, 31(4), 417-429.

Garduño-Monroy, V.H., Spinnler, J., Ceragioli, E., 1993, Geological and structural study of the Chapala rift, State of Jalisco, Mexico: Geofísica Internacional,32(3), 487-499.

Gasse, F., 1980, Les Diatomées Lacustres PlioPléistocénes du Gabeb (Ethiopie) Systematique, Paléoécologie, Biostratigraphie, 83p. 
Geiss, C.E., Zanner C.W., 2006, How abundant is pedogenic magnetite? Abundance and grain size estimates for loessic soils based on rock magnetic analyses: Journal of Geophysical Research, 111, B12S21. https://doi. org/10.1029/2006jb004564

Geiss C.E., Zanner C.W., Banerjee S.K., Minott J., 2004, Signature of magnetic enhancement in a loessic soil in Nebraska, United States of America: Earth and Planetary Science Letters, 228 (3/4), 355-367. https://doi. org/10.1016/j.epsl.2004.10.011

Geiss C.E., Egli R., Zanner C.W., 2008, Direct estimates of pedogenic magnetite as a tool to reconstruct past climatesfrom buried soils: Journal of Geophysical Research, 113, B11. http://doi.org/10.1029/2008JB005669

Germain, H., 1981, Flore des diatomées Diatomophycées- eaux douces et saumâtres du Massif Armoricain et des contrées voisines d'Europe occidentale Collection "Faunes et Flores Actuelles": Paris: Société Nouvelle des Editions Boubée, 444 p.

Guiry, M.D., Guiry, G.M., 2015, AlgaeBase. World-wide Electronic Publication, National University of Ireland, Galway.

Hunt C.P., Moskowitz B.M., Banerjee S.K., 1995, Magnetic properties of rocks and minerals, en Aherns T.J. (ed), Rock Physics and phase relations: A handbook of Physical Constants, Vol. 3: American Geophysical Union, 189204. http://doi.org/10.1029/RF003

Israde-Alcántara, I., Garduño-Monroy, Victor H.,1999, Lacustrine record in a volcanic intra-arc setting: The evolution of the Late Neogene Cuitzeo Basin System (Central Western Mexico, Michoacán: Paleogeography, Palaeoclimatology, Palaeoecology, 151(1-3), 209-227. https:// doi.org/10.1016/S0031-0182(99)00024-3

Israde-Alcántara, I., Garduño-Monroy, V.H., Fisher, C.T., Pollard, H.P., Rodríguez-Pascua M.A., 2005, Lake level change, climate, and the impact of natural events: the role of seismic and volcanic events in the formation of the Lake Patzcuaro Basin, Michoacan,
Mexico: Quaternary International, 135(1), 35-46. https://doi.org/10.1016/j. quaint.2004.10.022

Israde-Alcántara, I., Miller W.E., GarduñoMonroy V.H., Barron J., RodríguezPascua M.A., 2010a, Palaeoenvironmental significance of diatom and vertebrate fossils from Late Cenozoic tectonic basins in westcentral México: A review: Quaternary International, 219(1-2), 79-94. https://doi. org/10.1016/j.quaint.2010.01.012

Israde-Alcántara, I., Velázquez-Durán, R., Lozano-García, M., Bischoff, J., DomínguezVázquez, G., Garduño-Monroy, V. H., 2010b, Evolución Paleolimnológica del Lago de Cuitzeo, Michoacán durante el PleistocenoHoloceno: Boletín de la Sociedad Geológica Mexicana, 62(3), 345-357. https://doi. org/10.18268/bsgm2010v62n3a3

Jelonowski, A., Tucholka, P., Wiekowski, K., 1997, Magnetic properties of sediments in a Polish lake: evidence of a relation between the rockmagnetic record and environmental changes in Late Pleistocene and Holocene sediments: Geophysical Journal International, 129, 727-736. https://doi.org/10.1111/j.1365246X.1997.tb04508.x

Jordanova, N., Kovacheva, M., Hedley, I., Kostadinova, M., 2003, On the suitability of baked clay for archaeomagnetic studies as deduced from detailed rockmagnetic studies: Geophysical Journal International, 153, 146-158. https://doi. org/10.1046/j.1365-246X.2003.01889.x

Khursevich, G.K., Kociolek, J.P., Fedenya, S.A., 2002, A new genus of fossil freshwater diatoms (Bacillariophyta: Stephanodiscaceae) from the sediments of Lake Baikal: Proceedings of the California Academy of Sciences, 53, $1-10$.

Khursevich, G.K., Fedenya, S.A., Kuzmin, M.I., Karabanov, E.B., Williams, D.F., Prokopenko, A.A., 2003, Morphology of new taxa of Bacillariophyta from the Pliocene and Pleistocene deposits of Lake Baikal: International Journal on Algae, 5(3), 85-102. 
https://doi.org/10.1615/interjalgae.v5.i3.70

Khursevich, G., Kociolek, J.P., 2012, A preliminary, worldwide inventory of the extinct, freshwater fossil diatoms from the orders Thalassiosirales, Stephanodiscales, Paraliales, Aulacoseirales, Melosirales, Coscinodiscales, and Biddulphiales: Nova Hedwigia Beiheft, 141, 315-364.

Kirschvink, J. L., 1980, The least squares line and plane and the analysis of paleomagnetic data: Geophysical Journal International, 62(3), 699-718. https://doi.org/10.1111/j.1365246X.1980.tb02601.x

Kociolek, J.P., Laslandes, B., Bennett, D., Thomas, E., Brady, M., Graeff, C., 2014, Diatoms of the United States 1, Bibliotheca Diatomologica, Band 61, 188 p.

Krammer, K., 1980, Morphological and Taxonomic Investigations of Some Freshwater Species of the Diatom genus Amphora Ehr: Bacillaria, 6,197-226.

Krammer, K., Lange-Bertalot, H., 1985, Naviculaceae. Bibliotheca Diatomologia, Band 9. J. Cramer, Berlin-Stuttgart, 230 p.

Krammer, K. and Lange-Bertalot, H., 1986, Bacillariophyceae. 1. Teil: Naviculaceae. en Ettl, H., Gerloff, J., Heynig, H., Mollenhauer, D. (eds) Süsswasser flora von Mitteleuropa, Band 2/1. Gustav Fischer Verlag: Stuttgart, New York, 876 p.

Krammer, K., Lange-Bertalot, H., 1988, Bacillariophyceae. 2. Teil: Bacillariaceae, Epithemiaceae, Surirellaceae. in Ettl, H., Gerloff, J., Heynig, H. and Mollenhauer, D. (eds) Süsswasserflora von Mitteleuropa, Band 2/2. VEB Gustav Fischer Verlag: Jena, 596 p. Krammer, K., Lange-Bertalot, H., 1991a, Bacillariophyceae. 3. Teil: Centrales, Fragilariaceae, Eunotiaceae. in Ettl, H., Gerloff, J., Heynig, H. and Mollenhauer, D. (eds) Süsswasserflora von Mitteleuropa, Band 2/3. Gustav Fischer Verlag: Stuttgart, Jena, $576 \mathrm{p}$.

Krammer, K., Lange-Bertalot, H., 1991b, Bacillariophyceae. 4. Teil: Achnanthaceae, Kritische Ergänzungen zu Navicula
(Lineolatae) und Gomphonema, Gesamtliteraturverzeichnis Teil 1-4. in Ettl, H., Gärtner, G., Gerloff, J., Heynig, H. and Mollenhauer, D. (eds) Süsswasserflora von Mitteleuropa, Band 2/4. Gustav Fischer Verlag: Stuttgart, Jena, 437 p.

Lambeck, K., Rouby, H., Purcell, A., Sun, Y., Sambridge, M., 2014, Sea level and global ice volumes from the Last Glacial Maximum to the Holocene: Proceedings of the National Academy of Science of the USA, 111(43), 15296-15303. https://doi.org/10.1073/ pnas. 1411762111

Liu, Q., Deng, Ch., Torrent, J., Zhu, R., 2007, Review of recent developments in mineral magnetism of the Chinese loess: Quaternary Science Review, 26, 368-385. https://doi. org/10.1016/j.quascirev.2006.08.004

Maher B.A., 1986, Characterization of soils by mineral magnetic measurements: Physics of the Earth and Planetary Interiors, 42(1-2), 76-92. https://doi.org/10.1016/ S0031-9201(86)80010-3

Maher, B.A., Thompson, R., 1999, Quaternary climates, environments and magnetism: Cambridge, Cambridge University Press, 390 p. https://doi.org/10.1017/ CBO9780511535635

Mercer, L.T., Kowallis B.J., Christiansen, E.H., Miller, W.E., Carranza-Castañeda, O., Israde-Alcántara, I., 2014, Geology of the Tierras Blancas area in the southeastern part of the Acambay graben, central Mexico: The Geological Society of America Digital Map and Chart 18, 1-46 p. https://doi. org/10.1130/2014.dmch018

Metcalfe, S. E., 1992, Changing environments of the Zacapu basin, central Mexico: a diatom based history spanning the last 30,000 years, Research paper 48: Oxford, School of Geography.

Metcalfe, S.E., 1995, Holocene environmental change in the Zacapu Basin, Mexico: a diatom-based record: The Holocene, 5(2), 196-208. https://doi. org/10.1177\%2F095968369500500207 
Miller Wade, E., Carranza Castañeda, O., 1984, Late Cenozoic mammals from central Mexico: Journal of Vertebrate Paleontology, 4 (2), 216-236. https://www.tandfonline. $\mathrm{com} / \mathrm{doi} / \mathrm{abs} / 10.1080 / 02724634.1984 .100$ 12005

Miranda, J., Oliver, A., Vilaclara, G.,RicoMontiel, R., Macías, V.M., Ruvalcaba, J.L., Zenteno, M.A., 1994, Analysis of diatomite sediments from a paleolake in central Mexico using PIXE, X-ray tomography and X-ray diffraction: Nuclear Instruments and Methods in Physics Research Section B: Beam Interactions with Materials and Atoms, 85(1-4), 886-889. https://doi. org/10.1016/0168-583X(94)95943-9

Morales, J., Zarate del Valle, P., Pérez-Izazaga, E., Espinoza-Encinas, I., Velázquez-Bucio, M., Goguitchaichvili, A., Israde-Alcántara, I., 2019, Rock-magnetic and paleomagnetic study on a 27-m-long core from Lake Chapala, western Mexico: Paleoenvironmental implications for the last $10 \mathrm{ka}$ : Physics of the Earth and Planetary Interiors, 289, 90-105. https://doi.org/10.1016/j.pepi.2019.02.001

Moran, R.S., Clayton, L., 1984, Chronology of late Wisconsinan glaciation in Middle North America: Quaternary Science Reviews, 3(2-3), 1-6. https://doi. org/10.1016/0277-3791(84)90018-0

Mullins C.E., 1977, Magnetic susceptibility of the soil and its significance in soil science: A review: Journal Soil Science, 28(2), 223-246. https://doi.org/10.1111/j.1365-2389.1977. tb02232.x

Orgeira, M.J., Pereyra, F.X., Vásquez, C., Castañeda, E., Compagnucci, R., 2008, Rock magnetism in modern soils, Buenos Aires Province, Argentina: Journal of South American Earth Sciences, 26, 217-224. https://doi.org/10.1016/j. jsames.2008.03.007

Ortega Guerrero, B., Caballero Miranda, M., Lozano García, S., De la O-Villanueva, M., 1999, Palaeoenvironmental record of the last 70,000 yr in San Felipe Basin, Sonora desert,
México: Preliminary results: Geofísica Internacional, 38(3), 1-11.

Ortega Guerrero, B., Thompson, R., Urrutia Fucugauchi, J., 2000, Magnetic properties of lake sediments from Lake Chalco, central Mexico, and their paleoenvironmental implications: Journal of Quaternary Science, 15(2), 127-140. https://doi.org/10.1002/(SICI) 1099$1417(200002) 15: 2 \% 3 \mathrm{C} 127:$ :A ID JQS474\%3E3.0.CO;2-Z

Ortega, B., Caballero, C., Lozano, S., Israde, I., Vilaclara, G., 2002, 52000 years of environmental history in Zacapu basin, Michoacán, México: the magnetic record: Earth and Planetary Science Letters, 202, 663-675. https://doi.org/10.1016/ S0012-821X $(02) 00802-6$

Reimer, P.J., Bard, E., Bayliss, A., Beck, J.W., Blackwell, P.G., et al., 2013, IntCal13 and Marine13 radiocarbon age calibration curves 0-50,000 years cal BP: Radiocarbon, 55(4), 1869-1887. https://doi.org/10.2458/ azu_js_rc.55.16947

Rioual, P., Andrieu-Ponel, V., de Beaulieu, J.L., Reille, M., Svobodova, H., Battarbee, R.W., 2007, Diatom responses to limnological and climatic changes at Ribains Maar (French Massif Central) during the Eemian and Early Würm: Quaternary Science Reviews, 26, 1557-1609. https://doi.org/10.1016/j. quascirev.2007.03.009

Robles-Camacho, J., Corona-Chávez, P., Morales-Gámez, M., Guzmán, A.F., Polaco, Ó.J., Domínguez-Vázquez, G., IsradeAlcántara, I., Oliveros-Morales, A., 2010, Estratigrafía y paleoambiente asociados a un Gomphoteriidae (Cuvieronius hyodon) en Tzintzuntzan, Michoacán, México: Revista Mexicana de Ciencias Geológicas, 27(3), 530-544.

Rodríguez-Pascua, M.A., Garduño-Monroy, V.H., Israde-Alcántara, I., Pérez-López, R., 2010, Estimation of the paleoepicentral area from the spatial gradient of deformation in lacustrine seismites (Tierras Blancas Basin, 
Mexico): Quaternary International, 219, 66-78. https://doi.org/10.1016/j. quaint.2009.11.006

Roy, P. D., Charles-Polo, M.P., Lopez-Balbiaux, N., Pi-Puig, T., Sankar, G.M., LozanoSantacruz, R., Lozano-García, S., Romero F.M., 2014, Last glacial hydrological variations at the southern margin of subtropical North America and a regional comparison: Journal of Quaternary Science, 29(5). https://doi.org/10.1002/ jqs. 2718

Serieyssol, K., Gasse, F., 1991, Diatomees Neogenes du massif central Francais: quelques faits biostratigraphiques: Comptes rendus Academie des Sciences Paris, 312(2), 957-964.

Thompson, R., Oldfield, F., 1986, Environmental Magnetism, Allen and Unwin, Londres: Earth Surface Processes and Landforms, 13(1), 94-95. https://doi.org/10.1002/ esp.3290130115

Tite, M.S., Linington, R.E., 1975, Effect of climate on the magnetic susceptibility of soils: Nature, 256, 565-566. https://doi. org/10.1038/256565a0

Urrutia-Fucugauchi, J., Ortega-Ramírez, J., Cruz-Gatica, R., 1997, Rock-magnetic study of Late Pleistocene-Holocene sediments from the Babícora lacustrine basin, Chihuahua, northern Mexico: Geofísica Internacional, 36, 77-86.

Van Meerbeeck, C. J., Renssen, H., Roche, D.M., 2009, How did Marine Isotope Stage 3 and Last Glacial Maximum climates differ? Perspectives from equilibrium simulations:
Climate of the Past, 5(1), 33-51. https://doi. org/10.5194/cp-5-33-2009

Van Vugt, N., Steenbrink, J., Langereis, C.G., Hilgen, F.J., Meulenkamp, J.E., 1998, Magnetostratigraphy-based astronomical tuning of the early Pliocene lacustrine sediments of Ptolemais (NW Greece) and bed-tobed correlation with the marine record: Earth and Planetary Science Letters, 164, 535-551. https://doi.org/10.1016/ S0012-821X(98)00236-2

Vázquez, C. G., 2004, Caracterización de los sedimentos del Holoceno tardío del Lago de Santa María del Oro, Nayarit, utilizando métodos de magnetismo ambiental, Tesis de Maestría, Instituto de Geofísica, UNAM, 153 p.

Vázquez, G., Ortega, B., Davies, S.J., Aston, B.J., 2010, Registro sedimentario de los últimos 17000 años del lago de Zirahuén, Michoacán, México: Boletín de la Sociedad Geológica Mexicana, 3(62), 325-243. https://doi. org/10.18268/bsgm2010v62n3a2

Wang, L., Houyuan Lu, Jiaqi Liu, Zhaoyan Gu, Jens Mingram, Guoqiang Chu, Jingjing Li, Patrick Rioual, Jörg F.W. Negendank, Jingtai Han, Tungsheng Liu, 2008, Diatombased inference of variations in the strength of Asian winter monsoon winds between 17,500 and 6000 calendar years B.P: Journal of Geophisical Research, 113, D21. https:// doi.org/10.1029/2008JD010145

Williams, D., Round, F., 1987, Revision of the genus Fragilaria: Diatom Research, 2(2), 267-288. https://doi. org/10.1080/0269249X.1987.9705004 